\title{
Vector Penalty-Projection Methods for Open Boundary Conditions with Optimal Second-Order Accuracy
}

\author{
Philippe Angot ${ }^{1}$ and Rima Cheaytou ${ }^{1, *}$ \\ ${ }^{1}$ Aix-Marseille Université, Institut de Mathématiques de Marseille (I2M) - CNRS \\ UMR7373, Centrale Marseille - France.
}

Communicated by Kun Xu

Received 17 January 2018; Accepted (in revised version) 11 December 2018

\begin{abstract}
Recently, a new family of splitting methods, the so-called vector penaltyprojection methods (VPP) were introduced by Angot et al. [4,7] to compute the solution of unsteady incompressible fluid flows and to overcome most of the drawbacks of the usual incremental projection methods. Two different parameters are related to the VPP methods: the augmentation parameter $r \geq 0$ and the penalty parameter $0<\varepsilon \leq 1$. In this paper, we deal with the time-dependent incompressible Stokes equations with open boundary conditions using the VPP methods. The spatial discretization is based on the finite volume scheme on a Marker and Cells (MAC) staggered grid. Furthermore, two different second-order time discretization schemes are investigated: the second-order Backward Difference Formula (BDF2) known also as Gear's scheme and the Crank-Nicolson scheme. We show that the VPP methods provide a second-order convergence rate for both velocity and pressure in space and time even in the presence of open boundary conditions with small values of the augmentation parameter $r$ typically $0 \leq r \leq 1$ and a penalty parameter $\varepsilon$ small enough typically $\varepsilon=10^{-10}$. The resulting constraint on the discrete divergence of velocity is not exactly equal to zero but is satisfied approximately as $\mathcal{O}(\varepsilon \delta t)$ where $\varepsilon$ is the penalty parameter (taken as small as desired) and $\delta t$ is the time step. The choice $r=0$ requires special attention to avoid the accumulation of the round-off errors for very small values of $\varepsilon$. Indeed, it is important in this case to directly correct the pressure gradient by taking account of the velocity correction issued from the vector penalty-projection step. Finally, the efficiency and the second-order accuracy of the method are illustrated by several numerical test cases including homogeneous or non-homogeneous given traction on the boundary.
\end{abstract}

AMS subject classifications: 76D05, 76D07, 35Q35

Key words: Vector penalty-projection methods, Navier-Stokes equations, incompressible viscous flows, open boundary conditions, second-order accuracy.

*Corresponding author. Email addresses: philippe.angot@univ-amu.fr (P. Angot), rima. cheaytou@gmail.com (R. Cheaytou) 


\section{Introduction}

The numerical solution of incompressible flows has always been an important subject in fluid dynamics. The major difficulty in numerically solving unsteady incompressible Navier-Stokes equations in primitive variable form arises from the fact that the velocity and the pressure are coupled by the incompressibility constraint at each time step. There are numerous ways to discretize these equations, see e.g., the short review in [4]. Undoubtedly, the most popular are operator-splitting discretization schemes known as projection methods. This family of methods has been introduced by Chorin (1968) and Temam (1969) $[14,36]$. The interest in projection methods arises from the fact that the computations of the velocity and the pressure are decoupled by a two-step predictor-corrector procedure which significantly reduces the computational cost. In the first step, an intermediate velocity field is computed by solving momentum equations, ignoring the incompressibility constraint. In the second step, the predicted velocity field is projected onto a divergence-free vector field in order to get the pressure and the corrected velocity that satisfies the mass equation using the Helmholtz-Hodge decomposition. However, this process introduces a new numerical error, often named the splitting error, which must be at worst of the same order as the time discretization error. These projection methods were improved by Goda [18] in 1979 and named "the standard incremental projection methods"; they were popularized by Van Kan [38] in 1986 who introduced a second-order incremental pressure-correction scheme. It is well-known that in the projection step, a difficulty arises from the existence of an artificial pressure Neumann boundary condition which spoils the numerical solution of the pressure. This phenomenon was corrected by a variant proposed by Timmermans et al. [37] and analyzed by Guermond et al. [19] under the name "rotational incremental projection methods". A series of fractional step techniques including pressure-correction and incremental projection methods can be found in the review paper of Guermond et al. [20]. In 1992, Shen [35] introduced a modified approach which consists in adding a penalty term built from the divergence constraint in the first step of the scheme of the same form as in Augmented Lagrangian methods [17]. This approach is called "penalty-projection method". The same idea was suggested independently by Caltagirone and Breil [12] with some additional variants and was called "vector-projection step". In the same way, Jobelin et al. [30] proposed a numerical scheme which falls in the category of the penalty-projection method. This scheme generalizes the prediction step by an augmentation parameter totally independent of the time step and modifies consistently the projection step; numerical results using finite element approximation show that only small or moderate values of the augmentation parameter $r$ are sufficient to get accurate results. This numerical scheme was also theoretically analyzed in [35] and later in [8].

Recently, a new family of methods, the so-called "vector penalty-projection methods" (VPP) was proposed in [4]. Two parameters are related to the VPP methods: the augmentation parameter $r>0$ and the penalty-parameter $0<\varepsilon \leq 1$. These methods represent a compromise between the best properties of both classes: the Augmented Lagrangian 
methods (without inner iteration) and the splitting methods under a vector form. It was derived to overcome most of the drawbacks of the projection methods, see [4]: in fact, an original penalty-correction step for the velocity vector replaces the standard scalar pressure-correction to calculate flows with divergence-free velocity. These VPP methods are designed on the basis of both fast discrete Helmholtz-Hodge decompositions introduced in [9] and on the splitting penalty method proposed in [5] to efficiently solve general saddle-point problems. This allows us to easily impose the desired boundary condition to the end-of-step velocity pressure variables. The VPP methods were improved in $[1,6,7,13]$ where it is shown that such methods are also very efficient to compute incompressible multiphase viscous flows or Darcy flows whatever the density, viscosity or permeability jumps. Indeed, they are shown to favorably compete with the best incremental projection methods or Augmented Lagrangian methods in terms of accuracy, cheapness and robustness.

In $[1,4,7]$, the VPP methods were implemented using the first-order Euler implicit scheme in time with Dirichlet conditions on the boundary. The authors found that the scheme is $\mathcal{O}\left(h^{2}\right)$ in space for velocity and pressure, where $h$ is the spatial mesh step of the Marker and Cells (MAC) scheme and $\mathcal{O}(\delta t)$ in time for velocity and pressure ( $\delta t$ is the time step).

Many applications such as free surface problems and channel flows have to deal with open (traction or pseudo-traction) boundary conditions on a part of the boundary. In this paper, we are interested in the vector penalty-projection methods for open boundary conditions. The ability of projection methods to correctly treat open boundary conditions has been discussed in length in the literature. We report in this section some recent progress made in this direction.

Guermond et al. [20] use the standard incremental projection method and prove that the spatial convergence rate is between $\mathcal{O}(h)$ and $\mathcal{O}\left(h^{\frac{3}{2}}\right)$ for the velocity and $\mathcal{O}\left(h^{\frac{1}{2}}\right)$ for the pressure. They also obtain that the temporal convergence rate is between $\mathcal{O}(\delta \mathrm{t})$ and $\mathcal{O}\left(\delta t^{\frac{3}{2}}\right)$ for the velocity and $\mathcal{O}\left(\delta t^{\frac{1}{2}}\right)$ for the pressure. These results are improved by the rotational incremental scheme. The convergence rates for both velocity and pressure are expected to be between $\mathcal{O}(h)$ and $\mathcal{O}\left(h^{\frac{3}{2}}\right)$ in space and between $\mathcal{O}(\delta t)$ and $\mathcal{O}\left(\delta t^{\frac{3}{2}}\right)$ in time. Févrière et al. [16] combine the penalty-projection method with a spatial discretization by finite volume on staggered mesh. They obtain reasonably good results for moderate values of $r$ (typically $r=10$ ). These results are similar to those obtained with a finite element discretization [30]. Liu [32] presents a new numerical scheme using a pressure Poisson equation formulation and proposes new conditions for the pressure on the open or the traction boundaries. He proves the unconditional stability of a first-order semiimplicit scheme and shows second-order accuracy in time on velocity and pressure for the second-order scheme. Hasan et al. [23] present a new procedure for extrapolating velocities at the open boundary for the computations of incompressible flows around rigid bodies. Hosseini et al. [29] implement a rotational projection scheme to compute incompressible flows using Smoothed Particle Hydrodynamics (SPH). The scheme produces 
more accurate results especially for pressure and drag. It facilitates simulation with open boundaries and flow around solid obstacles. Poux et al. [33] propose a new numerical scheme in the framework of pressure-correction methods to compute the numerical solution of incompressible Navier-Stokes equations with open boundary conditions. They obtain good results both for spatial and temporal convergence rates. In particular, their method improves the standard incremental scheme to a spatial convergence of $\mathcal{O}\left(h^{2}\right)$ for velocity and pressure while remaining compatible with the rotational scheme. It also improves the orders of the standard incremental scheme to a temporal convergence rate of $\mathcal{O}\left(\delta t^{2}\right)$ for the velocity and close to $\mathcal{O}\left(\delta t^{2}\right)$ for pressure. Additionally, it slightly improves the orders of the rotational scheme to a convergence rate of $\mathcal{O}\left(\delta t^{2}\right)$ for velocity and pressure. For the same purpose, Poux et al. [34] have recently suggested a new numerical scheme in the framework of the velocity-correction methods with a proposed open boundary condition. They obtain good numerical results: concerning the spatial convergence, both the standard incremental and the rotational schemes lead to a second order convergence rate for velocity and pressure with the proposed open boundary conditions using the finite volume method. Concerning the temporal convergence, the rotational form of their method with the proposed open boundary condition improves the convergence rate to a second order convergence rate for velocity and pressure whereas it remains at $\mathcal{O}\left(\delta t^{\frac{3}{2}}\right)$ for velocity and $\mathcal{O}(\delta t)$ for pressure with the standard open boundary condition.

Finally, it is well-known that the Augmented Lagrangian method with Uzawa inner iterations, see e.g., [17], yields accurate results with Dirichlet or open boundary conditions, see e.g., [31]. However, this method suffers from locking effects when the augmentation parameter $r$ is large. In this case, many inner iterations are required and thus the solution cost is expensive, especially in 3 dimensions (3-D). For this reason, the splitting penalty methods proposed in [5] are very efficient by avoiding the locking effect with $r=1 / \varepsilon$ and for very small values of $\varepsilon$. The VPP methods presented in this paper are based on this splitting penalty method.

In the literature, the VPP methods concern only the case of the first-order time discretization with Dirichlet boundary conditions. The present paper is devoted to the extension of such methods to a second-order time discretization, either with the secondorder Backward Difference Formula (BDF2) or with the Crank-Nicolson scheme. Moreover, we study the case of open boundary conditions with a given traction. We propose two sets of open boundary conditions to naturally ensure the optimal second-order accuracy in both space and time through different benchmark problems. We believe that this paper provides an important progress since many formally second-order projection methods suffer from a degradation of precision when open boundary conditions are considered.

The objective of the present work is to show the optimal convergence rate of the vector-penalty projection methods when solving the unsteady incompressible viscous flow problems including open boundary conditions. We study both Stokes and NavierStokes problems since it is well-known that the degradation of accuracy also occurs for 
both linear and nonlinear viscous flows with most methods; see e.g. [20,21].

The remainder of the paper is organized as follows. In Section 2, the unsteady incompressible Navier-Stokes problem is stated and some notations are introduced. The VPP methods with Dirichlet boundary conditions are described in Section 3. We numerically justify the speed and the cheapness of the projection step in terms of iterations number while maintaining the penalty parameter $\varepsilon$ as small as possible. Section 4 is devoted to the presentation of the VPP methods with open boundary conditions using two different second-order schemes for time discretization: the BDF2 scheme and the Crank-Nicolson scheme. In addition, we draw a special attention to the choice of $r=0$ for small values of $\varepsilon$. In Section 5, various numerical results are presented, discussed and compared to the results obtained by other projection methods. We conclude in Section 6.

\section{Formulation of the continuous problem}

Let $\Omega \subset \mathbb{R}^{d}$ ( $d=2$ or 3 in practice) be an open, bounded and connected domain with a Lipschitz continuous boundary $\Gamma=\partial \Omega$. We suppose that $\Gamma$ is partitioned into two subsets $\Gamma_{D}$ and $\Gamma_{N}$, of outward unit normal vector $\mathbf{n}$, such that $\Gamma=\Gamma_{D} \cup \Gamma_{N}, \Gamma_{D} \cap \Gamma_{N}=\varnothing$. The generic point in $\Omega$ is denoted by $\mathbf{x}$.

We denote $L^{2}(\Omega)$-norm by $\|\cdot\|_{0}$, the $H^{1}(\Omega)$-norm by $\|\cdot\|_{1}$, the $H^{-1}(\Omega)$-norm by $\|\cdot\|_{-1}$ and $L^{2}(\Omega)$-inner product by $(., .)_{0}$.

Let us introduce the following functional spaces:

$$
\begin{aligned}
& \mathbf{L}^{2}(\Omega)=\left(L^{2}(\Omega)\right)^{d}, \\
& \mathbf{H}^{1}(\Omega)=\left\{\mathbf{u} \in \mathbf{L}^{2}(\Omega) ; \nabla u \in\left(L^{2}(\Omega)\right)^{d \times d}\right\}, \\
& \mathbf{W}(\Omega)=\left\{\mathbf{u} \in \mathbf{H}^{1}(\Omega)^{d} ; \mathbf{u}=0 \text { on } \Gamma_{D} \text { and } \mathbf{u} \cdot \mathbf{n}=0 \mathrm{on} \Gamma_{N}\right\}, \\
& L_{0}^{2}(\Omega)=\left\{q \in L^{2}(\Omega) ; \int_{\Omega} q d x=0\right\} .
\end{aligned}
$$

For $T>0$, we consider the time-dependent incompressible Navier-Stokes equations in the primitive variables on a finite time interval $[0, T]$ :

$$
\begin{aligned}
& \left.\rho\left(\frac{\partial \mathbf{v}}{\partial t}+(\mathbf{v} \cdot \nabla) \mathbf{v}\right)-\mu \Delta \mathbf{v}+\nabla p=\mathbf{f} \text { in } \Omega \times\right] 0, T[, \\
& \nabla \cdot \mathbf{v}=0 \quad \text { in } \Omega \times] 0, T[ \\
& \left.\mathbf{v}=\mathbf{v}_{D} \quad \text { on } \Gamma_{D} \times\right] 0, T[ \\
& \left.-p \mathbf{n}+\mu \nabla \mathbf{v} \cdot \mathbf{n}=\mathbf{g} \quad \text { on } \Gamma_{N} \times\right] 0, T[
\end{aligned}
$$

where $\mathbf{v}=(u, v)^{T}$ denotes the fluid velocity of initial value $\mathbf{v}(t=0)=\mathbf{v}_{0}, p$ the pressure field, $\rho$ the fluid density (the density is taken to 1 and $\mu$ the dynamic viscosity (here, $\mu=1 / R e$ with $R e$ a given Reynolds number). We impose Dirichlet boundary condition (2.3) on 
$\Gamma_{D}$ and a pseudo-traction condition (2.4) on $\Gamma_{N}$ which is derived from the pseudo-stress tensor $\widetilde{\sigma}$ given by:

$$
\widetilde{\sigma}=-p I+\mu \nabla \mathbf{v}
$$

The external body forces $\mathbf{f}$, the pseudo-stress vector $\mathbf{g}$ and the Dirichlet boundary condition $\mathbf{v}_{D}$ are known.

In this paper, we call (2.4) the open or natural boundary condition. In some situations, the force acting on $\Gamma_{N}$ might be given by

$$
\sigma(\mathbf{v}, p)=-p \mathbf{n}+\mu\left(\nabla \mathbf{v}+\nabla \mathbf{v}^{T}\right) \cdot \mathbf{n}=\mathbf{g}
$$

instead of (2.4).

Finally, the reader will keep in mind that bold letters such as $\mathbf{v}$, $\mathbf{g}$, etc., indicate vector valued quantities.

Remark 2.1 (Role of the pressure). In the incompressible flow, pressure is no longer a thermodynamic quantity but a Lagrange multiplier and it just establishes itself instantaneously in a flow field so that the velocity vector field always remains divergence free. Finally, we notice that the continuous Navier-Stokes problem does not need an initial condition for the pressure in the incompressible case and only an initial condition for the velocity is required.

\section{Vector penalty-projection methods with Dirichlet boundary conditions}

Let $0=t^{0}<t^{1}<\cdots<t^{N}=T$ be a partition of the time interval of computation $[0, \mathrm{~T}]$ which we suppose uniform for sake of simplicity. We denote by $\delta t=t^{n+1}-t^{n}>0$ the time step. Let $\phi^{0}, \phi^{1}, \cdots, \phi^{N}$ be a sequence of functions in a Hilbert space $\mathrm{H}$. We denote this sequence by $\phi_{\delta t}$ and we define the following discrete norm: $\left\|\phi_{\delta t}\right\|_{l^{2}(H)}:=\left(\delta t \Sigma_{n=0}^{N}\left\|\phi^{n}\right\|_{H}^{2}\right)^{1 / 2}$. The notation $\mathbf{v}^{n}$ is used to represent an approximation of $\mathbf{v}\left(t^{n}\right)$, where $t^{n}=n \delta t$.

\subsection{Description of the VPP methods with Dirichlet boundary conditions}

In this subsection, we present the vector penalty-projection methods for the incompressible Navier-Stokes problem supplemented with Dirichlet boundary conditions on the whole boundary $\Gamma$. Note that in this case, $\Gamma=\Gamma_{D}$ and $\Gamma_{N}=\varnothing$.

We use a semi-implicit time-integration scheme. We approximate the time derivative by the Backward Difference Formula of second-order (BDF2). The convective term is handled explicitly. Finally, the viscous term is treated implicitly. Hence, the VPP methods reads as follows.

Let $n \geq 1$ such that $(n+1) \delta t \leq T, \widetilde{\mathbf{v}}^{0}, \widetilde{\mathbf{v}}^{1}, \mathbf{v}^{0}, \mathbf{v}^{1} \in \mathbf{L}^{2}(\Omega)$ and $p^{0}, p^{1} \in L_{0}^{2}(\Omega)$ given. Find $\left(\mathbf{v}^{n+1}, p^{n+1}\right)$ such that: 
- Vector penalty-prediction step with an augmentation parameter $r \geq 0$ :

$$
\begin{aligned}
& \frac{3 \widetilde{\mathbf{v}}^{n+1}-4 \widetilde{\mathbf{v}}^{n}+\widetilde{\mathbf{v}}^{n-1}}{2 \delta t}+N L T_{1}-\mu \Delta \widetilde{\mathbf{v}}^{n+1}-r \nabla\left(\nabla \cdot \widetilde{\mathbf{v}}^{n+1}\right) \\
& \left.\quad+\nabla p^{\star, n+1}=\mathbf{f}^{n+1} \quad \text { in } \Omega \times\right] 0, T[, \\
& \left.\widetilde{\mathbf{v}}^{n+1}=\mathbf{v}_{D}^{n+1} \quad \text { on } \Gamma_{D} \times\right] 0, T[,
\end{aligned}
$$

where $p^{\star, n+1}$ is the second-order Richardson extrapolation for $p^{n+1}$ :

$$
p^{\star, n+1}=2 p^{n}-p^{n-1},
$$

and $N L T_{1}$ is the second-order extrapolated nonlinear term:

$$
N L T_{1}=2\left(\mathbf{v}^{n} \cdot \nabla\right) \widetilde{\mathbf{v}}^{n}-\left(\mathbf{v}^{n-1} \cdot \nabla\right) \widetilde{\mathbf{v}}^{n-1} .
$$

- Vector penalty-projection step with a penalty parameter $0<\varepsilon \leq 1$ :

$$
\begin{aligned}
& \frac{3 \widehat{\mathbf{v}}^{n+1}-4 \widehat{\mathbf{v}}^{n}+\widehat{\mathbf{v}}^{n-1}}{2 \delta t}+N L T_{2}-\varepsilon \mu \Delta \widehat{\mathbf{v}}^{n+1}-\frac{1}{\varepsilon} \nabla\left(\nabla \cdot \widehat{\mathbf{v}}^{n+1}\right) \\
& \left.\quad=\frac{1}{\varepsilon} \nabla\left(\nabla \cdot \widehat{\mathbf{v}}^{n+1}\right) \quad \text { in } \Omega \times\right] 0, T[, \\
& \left.\widehat{\mathbf{v}}^{n+1}=0 \quad \text { on } \Gamma_{D} \times\right] 0, T[,
\end{aligned}
$$

where $N L T_{2}$ is the second-order extrapolated nonlinear term:

$$
N L T_{2}=2\left(\mathbf{v}^{n} \cdot \nabla\right) \widehat{\mathbf{v}}^{n}-\left(\mathbf{v}^{n-1} \cdot \nabla\right) \widehat{\mathbf{v}}^{n-1} .
$$

- Correction step for velocity and pressure:

$$
\begin{aligned}
& \mathbf{v}^{n+1}=\widetilde{\mathbf{v}}^{n+1}+\widehat{\mathbf{v}}^{n+1}, \\
& p^{n+1}=2 p^{n}-p^{n-1}-\frac{1}{\varepsilon}\left(\nabla \cdot \mathbf{v}^{n+1}\right)-r \nabla \cdot \widetilde{\mathbf{v}}^{n+1} .
\end{aligned}
$$

At this stage, several comments can be made.

a. Using the second-order backward difference formula to discretize in time and the second-order extrapolation for the pressure and for the nonlinear terms leads to a formally 2nd-order scheme for both velocity and pressure.

b. Due to the explicit treatment of the nonlinear terms, the above scheme for nonlinear Navier-Stokes equations is subject to the usual CFL-like stability condition. We note that in order to ensure the unconditional stability of the semidiscretized system, a linearly implicit treatment of the nonlinear term can be also used, e.g. [6]. 
c. Contrary to the pressure-correction scheme in standard form, there is no artificial Neumann boundary condition imposed on the pressure approximation.

Remark 3.1 (Initial conditions for the pressure). It is useful to note that in the VPP method described above, we set $p^{0}=\left.p\right|_{t=0}$ which can be computed from the data and $p^{1}$ which can be computed by replacing BDF2 in the scheme above by the standard backward Euler formula as in [20,21].

Remark 3.2 (Nonlinear term in the projection step). Since the purpose of the velocity correction step is to perform an approximate divergence-free projection, it is not necessary to include the discretization of the nonlinear term in this step, see $[6,9]$. Thus, we can take $N L T_{2}=0$. In this case, it is more suitable to replace the nonlinear term $N L T_{1}$ in the prediction step by

$$
N L T_{1}=2\left(\mathbf{v}^{n} \cdot \nabla\right) \mathbf{v}^{n}-\left(\mathbf{v}^{n-1} \cdot \nabla\right) \mathbf{v}^{n-1},
$$

which is better for the consistency of the scheme.

Remark 3.3 (Stability and convergence of some numerical schemes for Navier-Stokes equations). Many works have been devoted to study the stability and convergence of many numerical schemes for solving the time-dependent Navier-Stokes equations. For example, see [27] for the Crank-Nicolson/Adams-Bashforth scheme, [26] for the semiimplicit second order Crank-Nicolson extrapolation scheme based on the stabilized finite element method, [24] for the two-level method based on finite element and the CrankNicolson extrapolation scheme, [28] for the Euler implicit/explicit scheme and [25] for a multilevel finite element method.

Remark 3.4 (Stability and convergence results for VPP methods). It is interesting to note that a rigorous theoretical study of the VPP methods with Dirichlet boundary conditions is provided in [3] where the authors show the stability of the scheme for $r \geq 0$ and prove that the method yields $\mathcal{O}\left(\delta t^{2}\right)$ accuracy for both the velocity and the pressure in the norm of $l^{\infty}\left(\mathbf{L}^{2}(\Omega)\right)$ and $l^{2}\left(L^{2}(\Omega)\right)$ respectively. Furthermore, we provide (in a submitted paper) a rigorous theoretical work concerning the VPP methods in the case of open boundary conditions where we show that the scheme is stable for $r \geq 0$ and we prove that the convergence rate of the error on the velocity and the pressure is of order 2 in $l^{\infty}\left(\mathbf{L}^{2}(\Omega)\right)$ and $l^{2}\left(L^{2}(\Omega)\right)$ respectively. Note that these theoretical results are in agreement with the numerical results.

\subsection{Space discretization and linear solvers}

In this section, we give a brief description of the space discretization and the tools used for the numerical simulations before presenting the numerical experiments. For the spatial discretization, the VPP method is implemented with a finite volume solver on the classical Marker and Cells grid (MAC mesh) of Harlow and Welch [22]. The MAC mesh is chosen for several reasons: it avoids the spurious modes of pressure, it does not need 
artificial boundary conditions. In our implementations, pressure unknowns are calculated at the cell-center and velocity components at mid-faces. All simulations presented are performed with a formally second-order scheme in time, i.e., a second-order Backward Difference Formula (BDF2) or the Crank-Nicolson scheme. Besides, the secondorder Richardson's extrapolation is used to extrapolate the pressure. Additionally, the method is initialized with a first time step performed with a standard backward Euler scheme. Finally, in order to solve the symmetric linear systems obtained in the prediction and projection steps, we are running the Conjugate Gradient (CG) method either with or without the zero-order Incomplete Cholesky $(\mathrm{IC}(0))$ as a preconditioner. The stopping criterion for the iterative (CG) method is chosen such that $\|$ res $\|_{2} \leq 10^{-6}$, where res denotes the residuals at the current CG iteration.

\subsection{Cost of the penalty-projection step}

In this part, we try to highlight the ambiguity that can interfere the reader regarding the perturbation of the viscous term in the second step by $\varepsilon$.

First, we write the implicit Euler scheme to discretize in time for sake of simplicity. Now, by focusing on the projection step, we explain below the interest to solve

$$
\begin{aligned}
& \frac{\widehat{\mathbf{v}}^{n+1}-\widehat{\mathbf{v}}^{n}}{\delta t}-\varepsilon \mu \Delta \widehat{\mathbf{v}}^{n+1}-\frac{1}{\varepsilon} \nabla\left(\nabla \cdot \widehat{\mathbf{v}}^{n+1}\right) \\
& \left.\quad=\frac{1}{\varepsilon} \nabla\left(\nabla \cdot \widetilde{\mathbf{v}}^{n+1}\right) \quad \text { in } \Omega \times\right] 0, T[\quad \text { (with perturbation), }
\end{aligned}
$$

instead of

$$
\begin{aligned}
& \frac{\widehat{\mathbf{v}}^{n+1}-\widehat{\mathbf{v}}^{n}}{\delta t}-\mu \Delta \widehat{\mathbf{v}}^{n+1}-\frac{1}{\varepsilon} \nabla\left(\nabla \cdot \widehat{\mathbf{v}}^{n+1}\right) \\
& \left.\quad=\frac{1}{\varepsilon} \nabla\left(\nabla \cdot \widetilde{\mathbf{v}}^{n+1}\right) \quad \text { in } \Omega \times\right] 0, T[\quad \text { (without perturbation). }
\end{aligned}
$$

We observe that the linear system associated to the projection step (with or without perturbation) can be solved all the more easily if $\eta=\varepsilon / \delta t$ is small enough. This is due to the fact that the operator in the right-hand side in the projection step is adapted to operator of the left-hand side. This leads to a fast and cheap vector correction step if $\eta=\varepsilon / \delta t$ is small enough as proved in [5, Theorem 1.1]. Moreover, the new projection step (3.7), where the viscous term is perturbed by $\varepsilon$, is much faster and cheaper if $\eta=\varepsilon / \delta t$ is sufficiently small as proved in [5, Corollary 1.3]. In order to verify these theoretical results in our case, we resort to a numerical test in which we compare the cost in terms of the number of iterations of the penalty-projection step of (3.8) and (3.7). The mesh size is fixed at $1 / h=128$, where $h$ is the spatial mesh step, and we run the algorithm for different values of $\varepsilon$ with a stationary tolerance set to $10^{-6}$ for Conjugate Gradient solvers. In order to solve the linear systems, we use and compare: 
- the standard Conjugate Gradient (CG) method;

- the Conjugate Gradient with IC(0) as a preconditioner (IC(0)-CG).

The representative curves of Fig. 1 show that the number of iterations decreases as long as $\varepsilon$ tends to zero. Moreover, in Fig. 1 (left), the cost of the projection step using (3.8) with standard (CG) method is about 400 iterations for $\varepsilon=10^{-10}$ whereas this cost is about 350 iterations for $\varepsilon=10^{-10}$ using (3.7). Furthermore, the cost of the projection step without perturbation (3.8) using the preconditioned solver (IC(0) - CG) (see Fig. 1 (right)) is decreased significantly, reaching 15 iterations for $\varepsilon=10^{-10}$. However, the number of iterations is reduced even more by using (3.7): only 4 iterations are required for $\varepsilon=10^{-10}$, i.e., about the quarter of the number of iterations obtained by the correction step (3.8) and this becomes quasi-independent of the spatial mesh step. These results are in agreement with the previous works $[4,5]$.
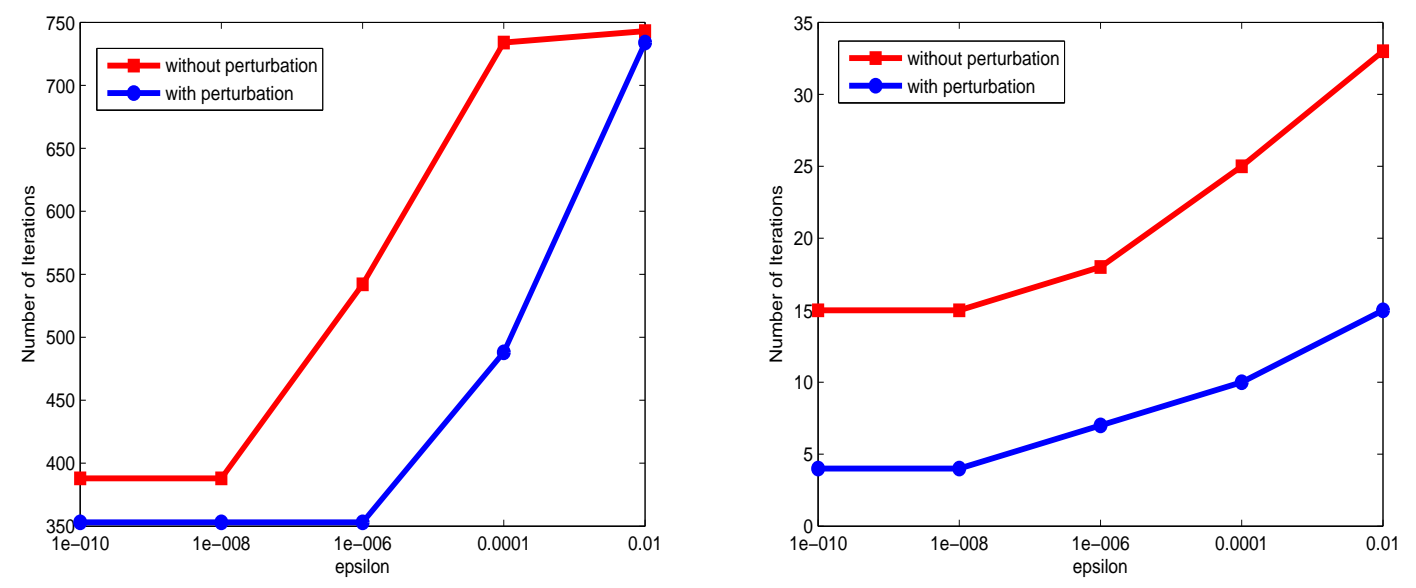

Figure 1: Cost of the penalty-projection step with perturbation (Eq. (3.7)) and without perturbation (Eq. (3.8)) at $T=2 \delta t$ with $\delta t=1$ and mesh size $1 / h=128$. Iterations number versus $\varepsilon$ using standard CG (left) and (IC(0)-CG) (right).

Indeed, we present in Fig. 2 the residual Euclidian norm of (3.7) with standard and preconditioned conjugate gradient iterations respectively. The result is clear: the convergence is improved by the preconditioner. Furthermore, we observe in Fig. 2 (left) that for the mesh size $16 \times 16$, the residual norm reaches approximately $10^{-8}$ for 100 iterations with standard CG. However, in Fig. 2 (right), it reaches $10^{-15}$ for the first iteration with preconditioned CG. Similarly, we found that for the mesh size $128 \times 128$, the residual norm is approximately of order $10^{-6}$ for 350 iterations with standard CG whereas using preconditioned (CG), the residual norm attains approximately $10^{-9}$ starting from 4 iterations. 

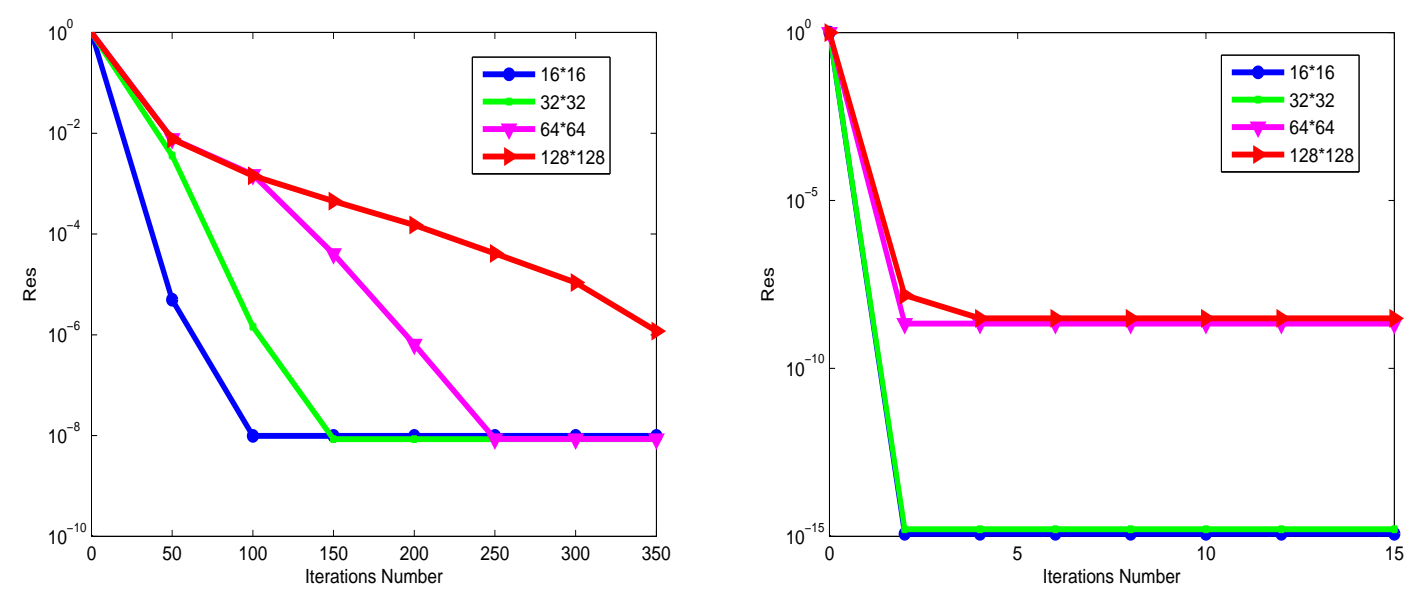

Figure 2: Normalized residual (by initial residual) versus number of iterations for different mesh sizes using Eq. (3.7) with standard CG (left) and (IC(0)-CG) (right) with $\eta=\varepsilon / \delta t=10^{-10}, T=2 \delta t$ and $\delta t=1$.

\section{Vector penalty-projection methods with open boundary conditions}

In many applications such as free surface problems and channel flows one has to deal with a natural boundary condition on the part $\Gamma_{N}$ of the border of the type:

$$
\left.(-p \mathbf{n}+\mu \nabla \mathbf{v} \cdot \mathbf{n})\right|_{\Gamma_{N}}=\mathbf{g} .
$$

Henceforth we assume that Dirichlet boundary condition is enforced on $\Gamma_{D}$ and an open boundary condition is enforced on $\Gamma_{N}$ where the whole boundary $\Gamma$ is defined as $\Gamma=\Gamma_{D} \cup \Gamma_{N}\left(\Gamma_{N} \neq \varnothing\right)$.

\subsection{Description of the $(V P P)$ methods with the first kind of open boundary condition OBC1 (see below) in the projection step}

We describe the VPP methods using OBC1 (see below) as an open boundary condition in the projection step with an augmentation parameter $r \geq 0$ and a penalty parameter $\varepsilon$ such that $0<\varepsilon \ll 1$. Let $\delta t>0$ be the time step. For the time discretization, we use in a first time the backward difference formula of second-order (BDF2) as in [2,30,31]. After that, we address the method using the Crank-Nicolson scheme $[15,24,26,27]$ which can be interpreted to be the average of the implicit and explicit Euler schemes.

\subsubsection{BDF2 time scheme}

Let $n \geq 1$ such that $(n+1) \delta t \leq T, \widetilde{\mathbf{v}}^{0}, \widetilde{\mathbf{v}}^{1}, \mathbf{v}^{0}, \mathbf{v}^{1} \in \mathbf{L}^{2}(\Omega)$ and $p^{0}, p^{1} \in L_{0}^{2}(\Omega)$ given. Find $\left(\mathbf{v}^{n+1}\right.$, $p^{n+1}$ ) such that: 
- Vector penalty-prediction step with an augmentation parameter $r \geq 0$ :

$$
\begin{aligned}
& \frac{3 \widetilde{\mathbf{v}}^{n+1}-4 \widetilde{\mathbf{v}}^{n}+\widetilde{\mathbf{v}}^{n-1}}{2 \delta t}-\mu \Delta \widetilde{\mathbf{v}}^{n+1}+N L T_{1}-r \nabla\left(\nabla \cdot \widetilde{\mathbf{v}}^{n+1}\right) \\
& \quad+\nabla p^{\star, n+1}=\mathbf{f}^{n+1} \quad \text { in } \Omega, \\
& \widetilde{\mathbf{v}}^{n+1}=\mathbf{v}_{D}^{n+1} \quad \text { on } \Gamma_{D}, \\
& \left(-p^{\star, n+1}+r \nabla \cdot \widetilde{\mathbf{v}}^{n+1}\right) \mathbf{n}+\mu \nabla \widetilde{\mathbf{v}}^{n+1} \cdot \mathbf{n}=\mathbf{g}^{n+1} \text { on } \Gamma_{N},
\end{aligned}
$$

where $p^{\star, n+1}$ is the second-order Richardson extrapolation for $p^{n+1}$ :

$$
p^{\star, n+1}=2 p^{n}-p^{n-1},
$$

and $N L T_{1}$ is the second-order extrapolated nonlinear term (see Remark 3.2):

$$
N L T_{1}=2\left(\mathbf{v}^{n} \cdot \nabla\right) \mathbf{v}^{n}-\left(\mathbf{v}^{n-1} \cdot \nabla\right) \mathbf{v}^{n-1} .
$$

- Vector penalty-projection step with a penalty parameter $0<\varepsilon \leq 1$ :

$$
\begin{aligned}
& \frac{3 \widehat{\mathbf{v}}^{n+1}-4 \widehat{\mathbf{v}}^{n}+\widehat{\mathbf{v}}^{n-1}}{2 \delta t}-\varepsilon \mu \Delta \widehat{\mathbf{v}}^{n+1}-\frac{1}{\varepsilon} \nabla\left(\nabla \cdot \widehat{\mathbf{v}}^{n+1}\right) \\
& \quad=\frac{1}{\varepsilon} \nabla\left(\nabla \cdot \widetilde{\mathbf{v}}^{n+1}\right) \quad \text { in } \Omega, \\
& \widehat{\mathbf{v}}^{n+1}=0 \quad \text { on } \Gamma_{D}, \\
& \mu \nabla \widehat{\mathbf{v}}^{n+1} \cdot \mathbf{n}=0 \quad \text { on } \Gamma_{N} \quad(\mathrm{OBC} 1) .
\end{aligned}
$$

- Correction step for velocity and pressure:

$$
\begin{aligned}
& \mathbf{v}^{n+1}=\widetilde{\mathbf{v}}^{n+1}+\widehat{\mathbf{v}}^{n+1}, \\
& p^{n+1}=2 p^{n}-p^{n-1}-\frac{1}{\varepsilon}\left(\nabla \cdot \mathbf{v}^{n+1}\right)-r \nabla \cdot \widetilde{\mathbf{v}}^{n+1} .
\end{aligned}
$$

Remark 4.1 (Effective discrete problem). Adding the prediction and projection steps gives the discrete problem which is effectively solved by the above splitting scheme:

$$
\begin{aligned}
& \frac{3 \mathbf{v}^{n+1}-4 \mathbf{v}^{n}+\mathbf{v}^{n-1}}{2 \delta t}+N L T_{1}-\mu\left(\Delta \widetilde{\mathbf{v}}^{n+1}+\varepsilon \Delta \widehat{\mathbf{v}}^{n+1}\right) \\
& \quad+\nabla p^{n+1}=\mathbf{f}^{n+1} \quad \text { in } \Omega, \\
& (\varepsilon \delta t) \frac{p^{n+1}-p^{\star, n+1}}{\delta t}+\nabla \cdot \mathbf{v}^{n+1}+r \varepsilon \nabla \cdot \widetilde{\mathbf{v}}^{n+1}=0 \text { in } \Omega, \\
& \mathbf{v}^{n+1}=\mathbf{v}_{D}^{n+1} \text { on } \Gamma_{D}, \\
& \left(-p^{\star, n+1}+r \nabla \cdot \widetilde{\mathbf{v}}^{n+1}\right) \mathbf{n}+\mu \nabla \mathbf{v}^{n+1} \cdot \mathbf{n}=\mathbf{g}^{n+1} \text { on } \Gamma_{N} .
\end{aligned}
$$


We note that the method has a weak lack of consistency compared to the $(V P P)$ method presented in [4]. This is due to the perturbation of the viscous term in the correction step (4.4) step by $\varepsilon$. Nevertheless, the method can be fast and very cheap if $\eta=\varepsilon / \delta t$ is sufficiently small. In fact, the right-hand side in the projection step lies in the range of the left-hand side as $\varepsilon$ is taken small enough. This crucial property was already shown theoretically in [5, Theorem 1.1 and Corollary 1.3] and in [6, Theorem 3.1] and also numerically confirmed in $[5,6,13]$. Finally, the vector penalty-projection step (4.4) is the key to get a cheap and fast method when $\varepsilon$ is sufficiently small.

Remark 4.2 (Another possible variant). In the prediction step (4.1), it is possible to replace the pressure gradient $\nabla p^{\star, n+1}$ by $\nabla p^{n}$ and then modify the pressure correction (4.8) consistently by using $p^{n}$ instead of $p^{\star, n+1}=2 p^{n}-p^{n-1}$. However, it is important to keep the discretization of the traction boundary condition (4.6) in the second-order using Richardson's extrapolation of pressure $p^{\star, n+1}$ in order to ensure the effective second-order accuracy in time of the method.

Remark 4.3. The initial condition on the velocity is $\mathbf{v}^{0}=\mathbf{v}_{0}$ with $\widetilde{\mathbf{v}}^{0}=\mathbf{v}^{0}=\mathbf{v}_{0}$ and $\widehat{\mathbf{v}}^{0}=0$. To start the second-order VPP scheme, we need $\mathbf{v}^{1}$ and $p^{1}$. For this reason, we first solve the VPP method using Euler scheme of first-order for a given $\mathbf{v}^{0}$ instead of the BDF2 (or $\mathrm{AB} / \mathrm{CN}$ ) scheme. This permits to calculate $\widetilde{\mathbf{v}}^{1}$ and $\widehat{\mathbf{v}}^{1}$ and consequently to find $\mathbf{v}^{1}$ and $p^{1}$.

\subsubsection{Correction of the pressure gradient for $r=0$ and small values of $\varepsilon$}

The augmentation parameter $r$ is kept constant and within small values in order to avoid to excessively degrade the conditioning of the linear system associated to the prediction step choosing $r=10^{-4}$. However, when $r=0$, we obtain the standard prediction step. In this case, we observe a poor convergence in time for velocity and pressure with very small values of $\varepsilon$ : see the numerical results in Section 5.1. In fact, this phenomenon is due to the cumulation of the round-off errors when $\varepsilon$ is relatively small. To improve the convergence rate, we hence reconstruct the pressure field itself very fast from its gradient. This idea was proposed in $[1,5,7]$ where the authors have observed that it is numerically far better to update the pressure gradient directly to avoid the effect of round-off errors when $\varepsilon$ is very small. In this regard, the updating of the pressure in our case is as follows. Starting from (4.8) and taking $r=0$ :

$$
p^{n+1}=2 p^{n}-p^{n-1}-\frac{1}{\varepsilon}\left(\nabla \cdot \mathbf{v}^{n+1}\right) .
$$

Taking the gradient of the above equation, we find

$$
\nabla p^{n+1}=2 \nabla p^{n}-\nabla p^{n-1}-\frac{1}{\varepsilon} \nabla\left(\nabla \cdot \mathbf{v}^{n+1}\right) .
$$

On the other hand, we have the following equality in the penalty-projection step:

$$
\frac{3 \widehat{\mathbf{v}}^{n+1}-4 \widehat{\mathbf{v}}^{n}+\widehat{\mathbf{v}}^{n-1}}{2 \delta t}-\varepsilon \mu \Delta \widehat{\mathbf{v}}^{n+1}=\frac{1}{\varepsilon} \nabla\left(\nabla \cdot \mathbf{v}^{n+1}\right) .
$$


Now, replacing the term $\frac{1}{\varepsilon} \nabla\left(\nabla \cdot \mathbf{v}^{n+1}\right)$ in (4.9) by the terms in the left side of the above equality. Then, the following estimation of the gradient of the pressure can be used directly for the pressure gradient correction:

$$
\nabla p^{n+1}=2 \nabla p^{n}-\nabla p^{n-1}-\frac{3 \widehat{\mathbf{v}}^{n+1}-4 \widehat{\mathbf{v}}^{n}+\widehat{\mathbf{v}}^{n-1}}{2 \delta t}+\varepsilon \mu \Delta \widehat{\mathbf{v}}^{n+1} .
$$

\subsubsection{Adams-Bashfort/Crank-Nicolson time scheme}

The set of equations is discretized in time using the Crank-Nicolson scheme at time $t^{n+\frac{1}{2}}$. However, since the continuity equation (2.2) should be satisfied at every time step, so it is always defined at time $t^{n+1}$. By definition, we have $z^{n+\frac{1}{2}}:=\frac{1}{2}\left(z^{n+1}+z^{n}\right)$.

Let $n \geq 1$ such that $(n+1) \delta t \leq T, \widetilde{\mathbf{v}}^{0}, \widetilde{\mathbf{v}}^{1}, \mathbf{v}^{0}, \mathbf{v}^{1} \in \mathbf{L}^{2}(\Omega)$ and $p^{0}, p^{1} \in L_{0}^{2}(\Omega)$ given. Find $\left(\mathbf{v}^{n+1}, p^{n+1}\right)$ such that:

- Vector penalty-prediction step with an augmentation parameter $r \geq 0$ :

$$
\begin{aligned}
& \frac{\widetilde{\mathbf{v}}^{n+1}-\widetilde{\mathbf{v}}^{n}}{\delta t}+N L T_{1}-\frac{\mu}{2} \Delta\left(\widetilde{\mathbf{v}}^{n+1}+\widetilde{\mathbf{v}}^{n}\right)-r \nabla\left(\nabla \cdot \widetilde{\mathbf{v}}^{n+1}\right) \\
& \quad+\nabla p^{\star, n+\frac{1}{2}}=f^{n+\frac{1}{2}} \quad \text { in } \Omega, \\
& \widetilde{\mathbf{v}}^{n+1}=\mathbf{v}_{D}^{n+1} \text { on } \Gamma_{D}, \\
& \left(-p^{\star, n+\frac{1}{2}}+\frac{r}{2} \nabla \cdot\left(\widetilde{\mathbf{v}}^{n+1}+\widetilde{\mathbf{v}}^{n}\right)\right) \mathbf{n}+\frac{\mu}{2} \nabla\left(\widetilde{\mathbf{v}}^{n+1}+\widetilde{\mathbf{v}}^{n}\right) \cdot \mathbf{n} \\
& \quad=\mathbf{g}^{n+\frac{1}{2}} \text { on } \Gamma_{N},
\end{aligned}
$$

where $p^{\star, n+\frac{1}{2}}$ is a linear combination of the pressures at the two previous time steps $t^{n}$ and $t^{n+1}$ :

$$
p^{\star, n+\frac{1}{2}}=\frac{3}{2} p^{n}-\frac{1}{2} p^{n-1}
$$

and $N L T_{1}$ is the second-order Adams-Bashfort extrapolated nonlinear term:

$$
N L T_{1}=\frac{3}{2}\left(\mathbf{v}^{n} \cdot \nabla\right) \mathbf{v}^{n}-\frac{1}{2}\left(\mathbf{v}^{n-1} \cdot \nabla\right) \mathbf{v}^{n-1} .
$$

- Vector penalty-projection step with a penalty parameter $0<\varepsilon \leq 1$ :

$$
\begin{aligned}
& \frac{\widehat{\mathbf{v}}^{n+1}-\widehat{\mathbf{v}}^{n}}{\delta t}-\frac{\varepsilon \mu}{2} \Delta\left(\widehat{\mathbf{v}}^{n+1}+\widehat{\mathbf{v}}^{n}\right)-\frac{1}{2 \varepsilon} \nabla\left(\nabla \cdot\left(\widehat{\mathbf{v}}^{n}+\widehat{\mathbf{v}}^{n+1}\right)\right) \\
& \quad=\frac{1}{2 \varepsilon} \nabla\left(\nabla \cdot\left(\widehat{\mathbf{v}}^{n+1}+\widehat{\mathbf{v}}^{n}\right)\right) \quad \text { in } \Omega, \\
& \widehat{\mathbf{v}}^{n+1}=0 \quad \text { on } \Gamma_{D \prime}, \\
& \frac{\mu}{2} \nabla\left(\widehat{\mathbf{v}}^{n+1}+\widehat{\mathbf{v}}^{n}\right) \cdot \mathbf{n}=0 \quad \text { on } \Gamma_{N} .
\end{aligned}
$$


- Correction step for velocity and pressure:

$$
\begin{aligned}
& \mathbf{v}^{n+1}=\widetilde{\mathbf{v}}^{n+1}+\widehat{\mathbf{v}}^{n+1}, \\
& p^{n+\frac{1}{2}}=\frac{3}{2} p^{n}-\frac{1}{2} p^{n-1}-\frac{1}{2 \varepsilon} \nabla \cdot\left(\mathbf{v}^{n+1}+\mathbf{v}^{n}\right)-r \nabla \cdot \widetilde{\mathbf{v}}^{n+1} .
\end{aligned}
$$

Remark 4.4. The expression of the pressure in (4.18) is approximated at time $t^{n+\frac{1}{2}}$. However, our goal is to find the pressure field at time $t^{n+1}$. We can switch to the pressure at time $t^{n+1}$ easily since by definition: $p^{n+\frac{1}{2}}=\frac{1}{2}\left(p^{n+1}+p^{n}\right)+\mathcal{O}\left(\delta t^{2}\right)$. Hence

$$
p^{n+1}=2 p^{n+\frac{1}{2}}-p^{n} .
$$

Replacing $p^{n+\frac{1}{2}}$ in (4.19) by the terms in the right side of (4.18), the approximation of the pressure at time $t^{n+1}$ yields

$$
p^{n+1}=2 p^{n}-p^{n-1}-\frac{1}{\varepsilon} \nabla \cdot\left(\mathbf{v}^{n+1}+\mathbf{v}^{n}\right)-2 r \nabla \cdot \widetilde{\mathbf{v}}^{n+1} .
$$

\subsection{Description of VPP methods with the second kind of open boundary condition $\mathrm{OBC} 2$ (see below) in the projection step}

The VPP methods with OBC1 (see (4.6)) yields good numerical results (see Section 5). However, we observe that the well-posedness of the penalty-projection step using OBC1 is not straightforward. Thus, we propose to replace OBC1 by another version of open boundary condition called OBC2 (see below) which clearly yields a well-posed penaltyprojection step (see Lemma 4.1).

\subsubsection{BDF2 time scheme}

Let $n \geq 1$ such that $(n+1) \delta t \leq T, \widetilde{\mathbf{v}}^{0}, \widetilde{\mathbf{v}}^{1}, \mathbf{v}^{0}, \mathbf{v}^{1} \in \mathbf{L}^{2}(\Omega)$ and $p^{0}, p^{1} \in L_{0}^{2}(\Omega)$ given. Find $\left(\mathbf{v}^{n+1}\right.$, $p^{n+1}$ ) such that:

- Vector penalty-prediction step with an augmentation parameter $r \geq 0$ :

$$
\begin{aligned}
& \frac{3 \widetilde{\mathbf{v}}^{n+1}-4 \widetilde{\mathbf{v}}^{n}+\widetilde{\mathbf{v}}^{n-1}}{2 \delta t}+N L T_{1}-\mu \Delta \widetilde{\mathbf{v}}^{n+1}-r \nabla\left(\nabla \cdot \widetilde{\mathbf{v}}^{n+1}\right) \\
& \quad+\nabla p^{\star, n+1}=\mathbf{f}^{n+1} \quad \text { in } \Omega, \\
& \widetilde{\mathbf{v}}^{n+1}=\mathbf{v}_{D} \quad \text { on } \Gamma_{D}, \\
& \left(-p^{\star, n+1}+r \nabla \cdot \widetilde{\mathbf{v}}^{n+1}\right) \mathbf{n}+\mu \nabla \widetilde{\mathbf{v}}^{n+1} \cdot \mathbf{n}=\mathbf{g}^{n+1} \text { on } \Gamma_{N},
\end{aligned}
$$

where $p^{\star, n+1}$ is the second-order Richardson extrapolation of $p^{n+1}$ :

$$
p^{\star, n+1}=2 p^{n}-p^{n-1},
$$

and $N L T_{1}$ is the second-order extrapolated nonlinear term:

$$
N L T_{1}=2\left(\mathbf{v}^{n} \cdot \nabla\right) \mathbf{v}^{n}-\left(\mathbf{v}^{n-1} \cdot \nabla\right) \mathbf{v}^{n-1} .
$$


- Vector penalty-projection step with a penalty parameter $0<\varepsilon \leq 1$ :

$$
\begin{aligned}
& \frac{3 \widehat{\mathbf{v}}^{n+1}-4 \widehat{\mathbf{v}}^{n}+\widehat{\mathbf{v}}^{n-1}}{2 \delta t}-\varepsilon \mu \Delta \widehat{\mathbf{v}}^{n+1}-\frac{1}{\varepsilon} \nabla\left(\nabla \cdot \widehat{\mathbf{v}}^{n+1}\right) \\
& \quad=\frac{1}{\varepsilon} \nabla\left(\nabla \cdot \widetilde{\mathbf{v}}^{n+1}\right) \text { in } \Omega, \\
& \widehat{\mathbf{v}}^{n+1}=0 \text { on } \Gamma_{D}, \\
& \widehat{\mathbf{v}}^{n+1} \cdot \mathbf{n}=0 \quad \text { and } \quad\left(\mu \nabla \widehat{\mathbf{v}}^{n+1} \cdot \mathbf{n}\right) \wedge \mathbf{n}=0 \quad \text { on } \Gamma_{N} \quad(\mathrm{OBC} 2) .
\end{aligned}
$$

- Correction step for velocity and reconstruction of the pressure from its gradient:

$$
\begin{aligned}
& \mathbf{v}^{n+1}=\widetilde{\mathbf{v}}^{n+1}+\widehat{\mathbf{v}}^{n+1}, \\
& \nabla p^{n+1}=2 \nabla p^{n}-\nabla p^{n-1}-\frac{3 \widehat{\mathbf{v}}^{n+1}-4 \widehat{\mathbf{v}}^{n}+\widehat{\mathbf{v}}^{n-1}}{2 \delta t}+\varepsilon \mu \Delta \widehat{\mathbf{v}}^{n+1}-r \nabla\left(\nabla \cdot \widetilde{\mathbf{v}}^{n+1}\right) .
\end{aligned}
$$

Remark 4.5. For any vector $\mathbf{u} \in \mathbb{R}^{d}$ defined on $\Gamma$, the tangential component $\left.\mathbf{u} \wedge \mathbf{n}\right|_{\Gamma}$ is defined by $\left.\mathbf{u} \wedge \mathbf{n}\right|_{\Gamma}=\left.\mathbf{u}_{\tau} \wedge \mathbf{n}\right|_{\Gamma}$ with $\mathbf{u}_{\tau}=\mathbf{u}-(\mathbf{u} \cdot \mathbf{n}) \mathbf{n}$. Thus, for $\mathrm{d}=2$, we simply have $\left.\mathbf{u} \wedge \mathbf{n}\right|_{\Gamma}=\mathbf{u} \cdot \boldsymbol{\tau}$, where $\boldsymbol{\tau}$ denotes the unit tangential vector on $\Gamma$.

Lemma 4.1 (Well-posedness of the projection step). For all $\widetilde{\boldsymbol{v}}^{n+1} \in \boldsymbol{H}^{1}(\Omega), \varepsilon>0$ and $\delta t>0$, there exists a unique solution $\widehat{\boldsymbol{v}}^{n+1} \in \boldsymbol{W}(\Omega)$ to the velocity-correction step (4.24)-(4.26) at each time step, where

$$
\boldsymbol{W}(\Omega)=\left\{\boldsymbol{u} \in \boldsymbol{H}^{1}(\Omega)^{d} ; \boldsymbol{u}=0 \text { on } \Gamma_{D} \text { and } \boldsymbol{u} \cdot \boldsymbol{n}=0 \text { on } \Gamma_{N}\right\}
$$

is an Hilbert space.

Proof. Starting from the continuous formulation of the vector penalty-projection step:

$$
\frac{\partial \widehat{\mathbf{v}}}{\partial t}-\varepsilon \mu \Delta \widehat{\mathbf{v}}-\frac{1}{\varepsilon} \nabla(\nabla \cdot \widehat{\mathbf{v}})=\frac{1}{\varepsilon} \nabla(\nabla \cdot \widetilde{\mathbf{v}}) .
$$

Taking the inner product of the above equation with a test function $\varphi \in \mathbf{W}(\Omega)$ and applying Green's formula. This yields:

$$
\begin{gathered}
\int_{\Omega} \frac{\partial \widehat{\mathbf{v}}}{\partial t} \cdot \boldsymbol{\varphi} d \mathbf{x}+\varepsilon \int_{\Omega} \mu \nabla \widehat{\mathbf{v}}: \nabla \boldsymbol{\varphi} d \mathbf{x}-\varepsilon \int_{\Gamma}(\mu \nabla \widehat{\mathbf{v}} \cdot \mathbf{n}) \cdot \boldsymbol{\varphi} d s \\
+\frac{1}{\varepsilon} \int_{\Omega}(\nabla \cdot \widehat{\mathbf{v}})(\nabla \cdot \boldsymbol{\varphi}) d \mathbf{x}-\frac{1}{\varepsilon} \int_{\Gamma}(\nabla \cdot \widehat{\mathbf{v}})(\boldsymbol{\varphi} \cdot \mathbf{n}) d s \\
=-\frac{1}{\varepsilon} \int_{\Omega}(\nabla \cdot \widetilde{\mathbf{v}})(\nabla \cdot \boldsymbol{\varphi}) d \mathbf{x}+\frac{1}{\varepsilon} \int_{\Gamma}(\nabla \cdot \widetilde{\mathbf{v}})(\boldsymbol{\varphi} \cdot \mathbf{n}) d s .
\end{gathered}
$$


Since $\Gamma=\Gamma_{D} \cup \Gamma_{N}$ and by the fact that $\boldsymbol{\varphi}=0$ on $\Gamma_{D}, \boldsymbol{\varphi} \cdot \mathbf{n}=0$ on $\Gamma_{N}$ and $(\mu \nabla \widehat{\mathbf{v}} \cdot \mathbf{n}) \wedge \mathbf{n}=0$ on $\Gamma_{N}$, we obtain the following weak form:

$$
\begin{aligned}
& \int_{\Omega}\left(\frac{\partial \widehat{\mathbf{v}}}{\partial t} \cdot \boldsymbol{\varphi}+\varepsilon \mu \nabla \widehat{\mathbf{v}}: \nabla \boldsymbol{\varphi}+\frac{1}{\varepsilon}(\nabla \cdot \widehat{\mathbf{v}})(\nabla \cdot \boldsymbol{\varphi})\right) d \mathbf{x} \\
= & -\frac{1}{\varepsilon} \int_{\Omega}(\nabla \cdot \widetilde{\mathbf{v}})(\nabla \cdot \boldsymbol{\varphi}) d \mathbf{x} .
\end{aligned}
$$

For sake of simplicity, we take the discrete form of (4.30) and we use the implicit Euler scheme to discretize in time. We obtain the following bilinear form:

$$
a(\widehat{\mathbf{v}}, \boldsymbol{\varphi})=\frac{\varepsilon}{\delta t}(\widehat{\mathbf{v}}, \boldsymbol{\varphi})_{0}+\varepsilon^{2} \mu(\nabla \widehat{\mathbf{v}}, \nabla \boldsymbol{\varphi})_{0}+(\nabla \cdot \widehat{\mathbf{v}}, \nabla \cdot \boldsymbol{\varphi})_{0} .
$$

It is clear that $a(\widehat{\mathbf{v}}, \boldsymbol{\varphi})$ is a continuous and coercive form in $\mathbf{W}(\Omega) \times \mathbf{W}(\Omega)$. Moreover,

$$
L(\boldsymbol{\varphi}):=(\nabla \cdot \widetilde{\mathbf{v}}, \nabla \cdot \boldsymbol{\varphi})_{0}+\frac{\varepsilon}{\delta t}(\widehat{\mathbf{v}}, \varphi)_{0}
$$

is a linear continuous form in $\mathbf{W}(\Omega)$. Under these hypotheses, we can easily apply the Lax-Milgram theorem which ensures that the penalty-projection step (4.24)-(4.26) is wellposed and admits a unique solution $\widehat{\mathbf{v}}^{n+1}$ in the Hilbert space $\mathbf{W}(\Omega)$ equipped with the usual norm of $\mathbf{H}^{1}(\Omega)$ (as a closed subspace of $\mathbf{H}^{1}(\Omega)$ ).

Remark 4.6 (Third set of open boundary condition). We propose a new set of open boundary condition which we call (OBC3) in the projection step. To introduce the VPP methods with (OBC3), we proceed as follows. In the prediction step, there are no modifications to make. The step is exactly the same as proposed in VPP with (OBC2):

$$
\begin{aligned}
& \frac{3 \widetilde{\mathbf{v}}^{n+1}-4 \widetilde{\mathbf{v}}^{n}+\widetilde{\mathbf{v}}^{n-1}}{2 \delta t}+N L T_{1}-\mu \Delta \widetilde{\mathbf{v}}^{n+1}-r \nabla\left(\nabla \cdot \widetilde{\mathbf{v}}^{n+1}\right) \\
& \quad+\nabla p^{\star, n+1}=\mathbf{f}^{n+1} \quad \text { in } \Omega, \\
& \widetilde{\mathbf{v}}^{n+1}=\mathbf{v}_{D} \quad \text { on } \Gamma_{D}, \\
& \left(-p^{\star, n+1}+r \nabla \cdot \widetilde{\mathbf{v}}^{n+1}\right) \mathbf{n}+\mu \nabla \widetilde{\mathbf{v}}^{n+1} \cdot \mathbf{n}=\mathbf{g}^{n+1} \text { on } \Gamma_{N} .
\end{aligned}
$$

For the projection step, we define the proposed open boundary condition (OBC3) on $\Gamma_{N}$ as

$$
\begin{aligned}
& \frac{3 \widehat{\mathbf{v}}^{n+1}-4 \widehat{\mathbf{v}}^{n}+\widehat{\mathbf{v}}^{n-1}}{2 \delta t}-\mu \Delta \widehat{\mathbf{v}}^{n+1}-\frac{1}{\varepsilon} \nabla\left(\nabla \cdot \widehat{\mathbf{v}}^{n+1}\right) \\
& \quad=\frac{1}{\varepsilon} \nabla\left(\nabla \cdot \widehat{\mathbf{v}}^{n+1}\right) \quad \text { in } \Omega, \\
& \widehat{\mathbf{v}}^{n+1}=0 \quad \text { on } \Gamma_{D}, \\
& \mu \nabla \widehat{\mathbf{v}}^{n+1} \cdot \mathbf{n}+\frac{1}{\varepsilon} \nabla \cdot \mathbf{v}^{n+1}=0 \quad \text { on } \Gamma_{N} \quad(\mathrm{OBC} 3) .
\end{aligned}
$$


We numerically test this scheme with a formally second-order in time. We roughly observe a second order convergence rate in time for both the velocity and the pressure gradient (for case $r=0$ ). Moreover, a theoretical study including the well-posedness (see Lemma. 4.1), the stability and the convergence analysis has been established. The theoretical results are in line with the numerical ones. These results are the subject of a work in preparation.

Remark 4.7 (Theoretical analysis of VPP methods). The penalty-projection step in the present methods is based on the fast discrete Helmholtz-Hodge decompositions of $\mathbf{L}^{2}(\Omega)$ vector fields proposed in [9] for bounded domains. Some theoretical results of stability and error estimates are given for the first-order version of VPP methods with Dirichlet boundary conditions in $[4,5]$. The proof of stability for Navier-Stokes problems with such methods is stated in [1]. Moreover, the analogous continuous version of the VPP methods, the so-called two-step artificial compressibility method, is analyzed in [11]. In this case, the solutions are proved to converge to weak solutions of the Navier-Stokes equations when the penalty parameter $\varepsilon$ tends to zero.

In the case of open boundary conditions, it is stated that the Stokes problem with the stress (or traction) vector given on the boundary is globally well-posed whatever the dimension $d$. For the Navier-Stokes system, it is not at all clear that this boundary condition guarantees global existence and uniqueness of weak solutions without any restriction on the data, even in two dimensions. More precisely, it is only possible to prove, either global existence for a small Reynolds number (that is a quasi Stokes regime), or local existence with a small time interval $T$. Therefore, it is probably necessary to consider nonlinear boundary conditions which ensure the control of the kinetic energy at the artificial boundary. For example, we refer the reader to the artificial boundary condition taking account of the local inflow / open volume rate produced by a singular load, which is recently proposed in [10]. Indeed, Angot's open boundary condition for the NavierStokes equations leads to global existence of weak solutions in 3-D and uniqueness in $2-D$, as for the case of Dirichlet boundary conditions.

\section{Numerical experiments}

This section is organized in the following way. First, we focus on the behavior of the spatial and temporal convergence rates in the case of open boundary conditions (homogeneous and nonhomogeneous). The numerical tests include the Stokes and the NavierStokes problem. In addition, we estimate the $L^{2}$-norm of the velocity divergence. Finally, we conduct a comparative and qualitative study of the VPP methods presented in this paper and some pressure-correction schemes used in the literature for the solution of non-stationary incompressible flow problems, see, e.g., [20,33]. Finally, we note that for the numerical results concerning the case of Dirichlet condition on the whole boundary, we refer to [3] where we have shown numerically as well as theoretically that the convergence rate for the velocity and the pressure exhibits a second-order convergence rate in time. 


\subsection{Stokes flow with open boundary conditions}

We consider the square domain $\Omega=] 0,1[2$ and we enforce Dirichlet condition on $\Gamma=$ $\partial \Omega$ except for the part included in the y-axis, where open boundary conditions (2.4) are imposed. In this section, we illustrate the convergence properties of the VPP methods for two manufactured test cases with open boundary conditions, homogeneous or not.

\subsubsection{Homogeneous open boundary conditions $(\mathbf{g}=0)$}

We choose a test case already used in the literature [20,21]. It consists of unstationary Stokes problem, with a forcing term, an initial condition and boundary conditions corresponding to the following analytical solutions.

$$
\begin{aligned}
& u(x, y, t)=\sin (x) \sin (y+t), \\
& v(x, y, t)=\cos (x) \cos (y+t), \\
& p(x, y, t)=\cos (x) \sin (y+t) .
\end{aligned}
$$

Convergence rate in space. Since an optimal space convergence rate can be reached using projection methods in the case of Dirichlet boundary conditions, we are interested to study the space convergence rate for Stokes equations with open boundary conditions which is a more sensitive case. In order to estimate the spatial error, we focus on the stationary solution of the above numerical experiment. We take the time step $\delta t=10^{-2}$, the penalty parameter $\varepsilon=10^{-10}$, the augmentation parameter $r=10^{-4}$, and we run the algorithm for different values of the mesh space $h$. For more precision, we note that we test the spatial convergence using VPP methods with OBC1.

In Fig. 3, the convergence rate of the error on the velocity is clearly $\mathcal{O}\left(h^{2}\right)$ while the convergence rate in space for the pressure is around $\mathcal{O}\left(h^{2}\right)$.

As a conclusion concerning the spatial convergence rate, we observe that the results obtained here conform with those reported by Poux et al. [33]. In addition, the optimal convergence rate in space offered by VPP methods is also in concordance with the results obtained by [34] in the framework of the velocity-correction methods. Besides, the VPP method appears more efficient and accurate than the standard incremental scheme in [20]; particularly, it improves the spatial convergence from $\mathcal{O}(h)$ to $\mathcal{O}\left(h^{2}\right)$ for the velocity and from $\mathcal{O}\left(h^{1 / 2}\right)$ to $\mathcal{O}\left(h^{2}\right)$ for the pressure.

Convergence rate in time. In order to study the accuracy in time in the presence of open boundary conditions, we perform convergence tests with respect to $\delta t$. We consider the unsteady homogeneous case. In all the following tests, the mesh is chosen fine enough $(128 \times 128)$ to ensure that the consistency error in space is significantly smaller than the one in time.

As one can see in Fig. 4, the convergence rate of the error for the velocity behaves like $\mathcal{O}\left(\delta t^{2}\right)$ and the one for the pressure is also like $\mathcal{O}\left(\delta t^{2}\right)$ for different values of the augmentation parameter $r$ between $10^{-4}$ and 1 . These rates are similar to those obtained 

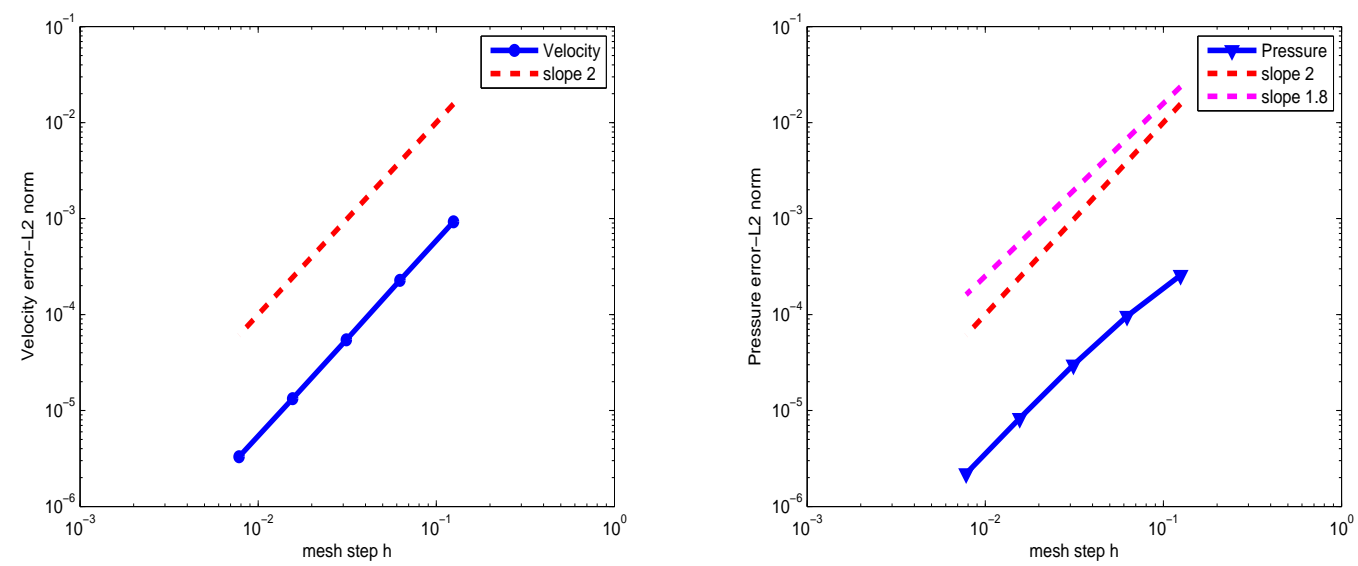

Figure 3: Homogeneous open boundary conditions. OBC1 - Spatial convergence rates for the velocity (left) and the pressure (right) at $T=2, \delta t=10^{-2}, \varepsilon=10^{-10}$ and $r=10^{-4}$.

by Poux et al. [33] for the rotational form of their method proposed in the framework of pressure-correction. Furthermore, in [34], the authors obtain an optimal convergence rate in the framework of the velocity-correction method (in standard incremental and rotational form) with a proposed open boundary condition. Their results are also in agreement with what we obtained here by the VPP methods. On the other hand, the errors of velocity and pressure for the VPP methods are smaller than those computed in [33] even if the mesh we have used is coarser. Moreover, the VPP methods improve the convergence rates of the standard BDF2 pressure-correction scheme from $\mathcal{O}(\delta t)$ to $\mathcal{O}\left(\delta t^{2}\right)$ for the velocity and from $\mathcal{O}\left(\delta t^{1 / 2}\right)$ to $\mathcal{O}\left(\delta t^{2}\right)$ for the pressure [19]. In [19], the second-order rotational pressure-correction yields $\mathcal{O}\left(\delta t^{3 / 2}\right)$ accuracy for the velocity in the $L^{2}$-norm and $\mathcal{O}(\delta t)$ accuracy for the pressure.

Fig. 5 illustrates the errors of the $L^{2}$-norm of the velocity divergence as a function of the penalty parameter $\varepsilon$ and the time step $\delta t$. In Fig. 5 (left), the $L^{2}$-norm of the divergence of the velocity vanishes as $\mathcal{O}(\varepsilon \delta t)$ when the penalty parameter $\varepsilon$ is chosen as small as desired. In Fig. 5 (right), the $L^{2}$-norm of the velocity divergence is around $\mathcal{O}(\varepsilon \delta t)$ for $\varepsilon$ small enough.

As a conclusion on the temporal convergence using VPP methods with OBC1, we notice that the convergence rate in the presence of open boundary conditions is brought to the level observed with the Dirichlet boundary conditions in [3] and guarantees a second-order accuracy in time for velocity and pressure. Moreover, the $L^{2}$-norm of the divergence of the velocity vanishes as $\mathcal{O}(\varepsilon \delta t)$ with a penalty parameter $\varepsilon$ too small.

Remark 5.1. We emphasize that the pseudo-stress tensor is commonly used in this kind of numerical experiments (see $[20,21,32,34]$ ). This is why we choose to restrict our test cases using the pseudo-traction boundary condition (2.4) to compare our results with those obtained by these previous works. 

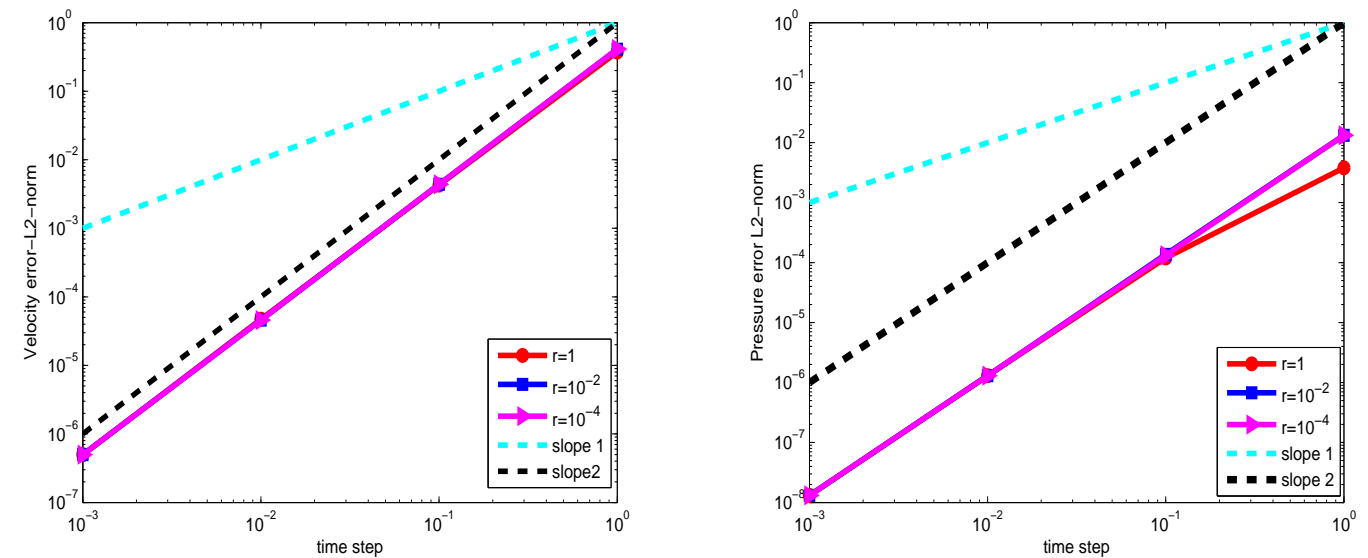

Figure 4: Homogeneous open boundary conditions. OBC1 - Temporal convergence rates for the velocity (left) and the pressure (right) at $T=2$, mesh size $1 / h=128$ and $\varepsilon=10^{-10}$.
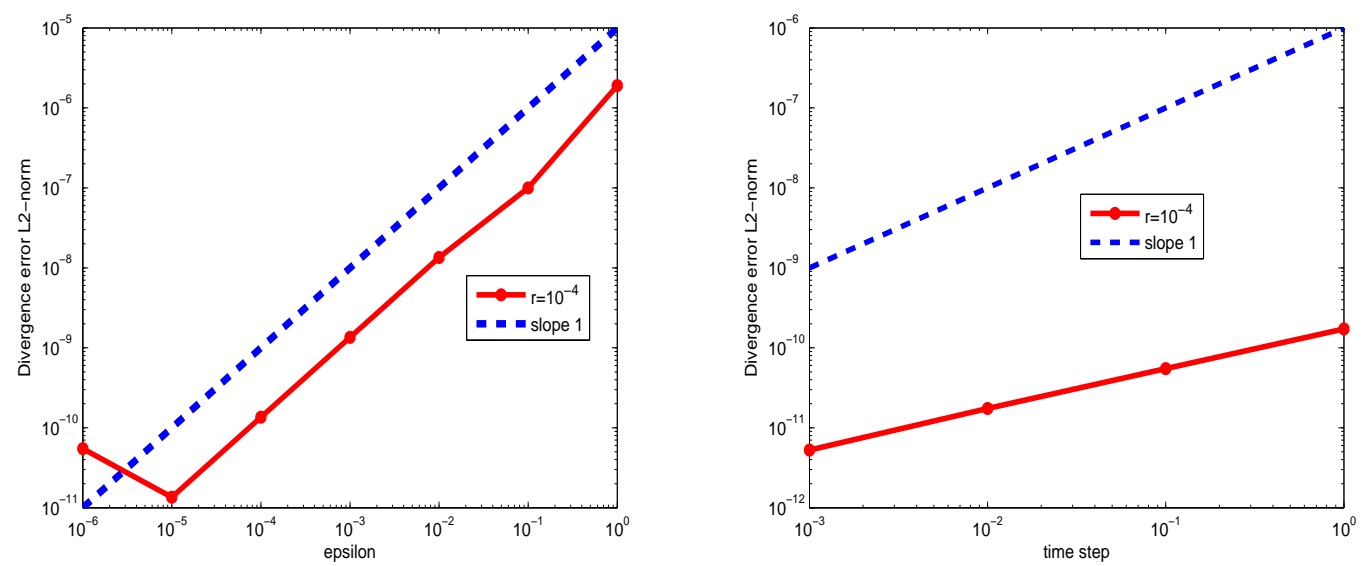

Figure 5: Homogeneous open boundary conditions. OBC1 - Velocity divergence $L^{2}$-norm versus $\varepsilon$ (left) at $T=2$, mesh size $1 / h=128$ and $r=10^{-4}$ - Velocity divergence $L^{2}$-norm versus time step (right) at $T=2$, mesh size $1 / h=128$ and $\varepsilon=10^{-6}$.

To complete the study, we have performed convergence tests for the VPP methods using the Crank-Nicolson scheme to approximate in time using the same analytical solution described above. We recall that the Crank-Nicolson scheme is also a second-order scheme in time.

Fig. 6 represents the $L^{2}$-norm of the error on the velocity and the pressure respectively as a function of the time step $\delta t$. The results of the error exhibit approximately a secondorder convergence rate for both unknowns. In addition, the slopes for both the velocity and the pressure error obtained by using the Crank-Nicolson scheme are slightly lower 

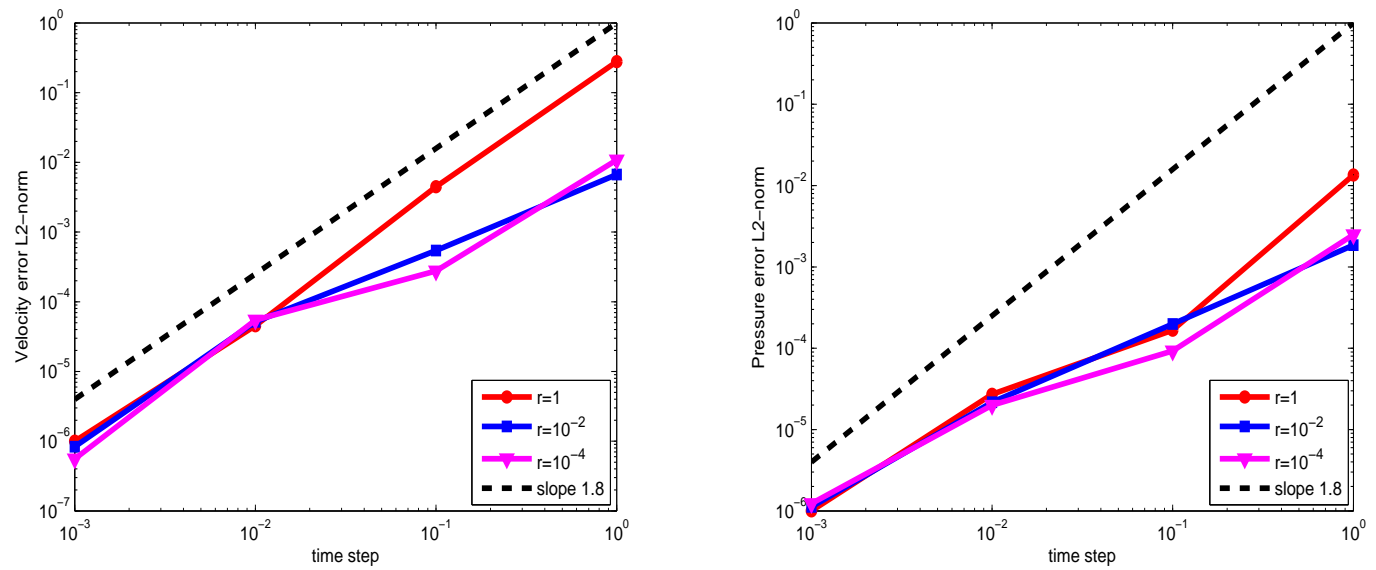

Figure 6: Homogeneous open boundary conditions. Crank-Nicolson - Temporal convergence rates for the velocity (left) and the pressure (right) at $T=2$, mesh size $1 / h=128$ and $\varepsilon=10^{-10}$.
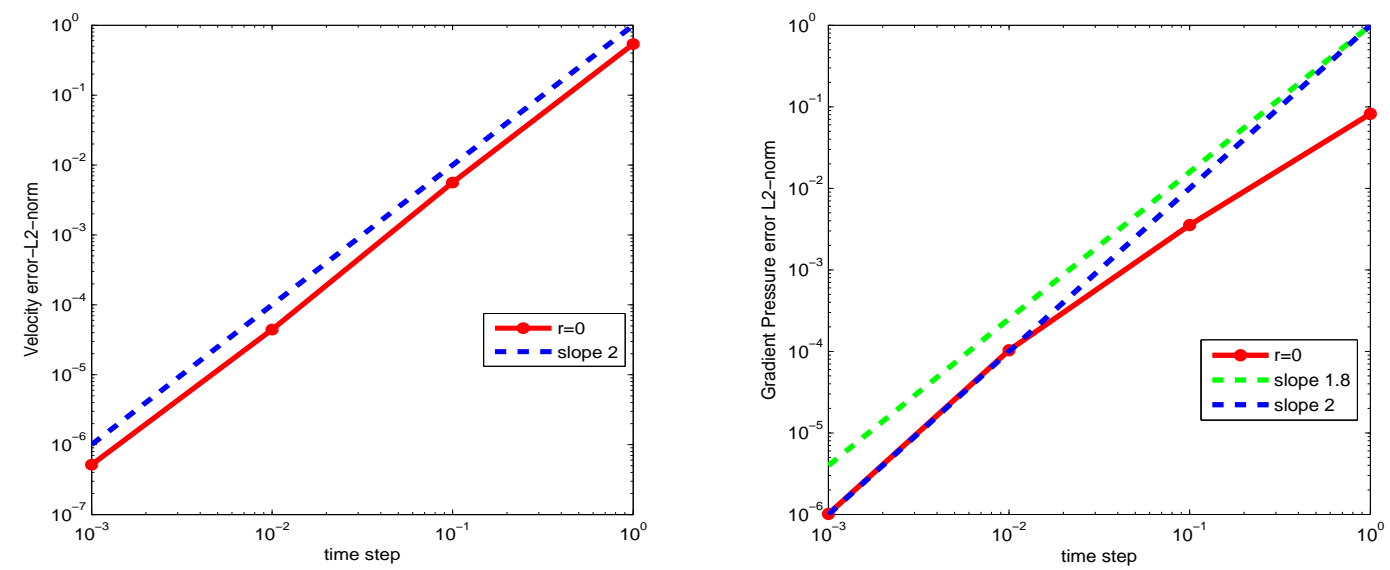

Figure 7: Homogeneous open boundary conditions. OBC2 - Temporal convergence rates for the velocity (left) and the gradient pressure (right) at $T=2$, mesh size $1 / h=128, \varepsilon=10^{-10}$ and $r=0$.

than the slopes obtained by using the BDF2-scheme. We also obtain an order of $\mathcal{O}(\varepsilon \delta t)$ for the $L^{2}$-norm of the velocity divergence.We did not show the figures for sake of shortness.

Finally, in order to check the VPP methods with the OBC2 (4.26) in the projection step, we numerically simulate the test case presented above. Fig. 7 displays the errors of the computed velocity and pressure gradient in the $L^{2}$-norm at $\varepsilon=10^{-10}$ and $r=0$. The numerical results show that a second-order accuracy in time is recovered for both the velocity and the pressure gradient. The slopes in Fig. 8 exhibit again that the $L^{2}$-norm of the velocity divergence is of order $\mathcal{O}(\varepsilon \delta t)$. 

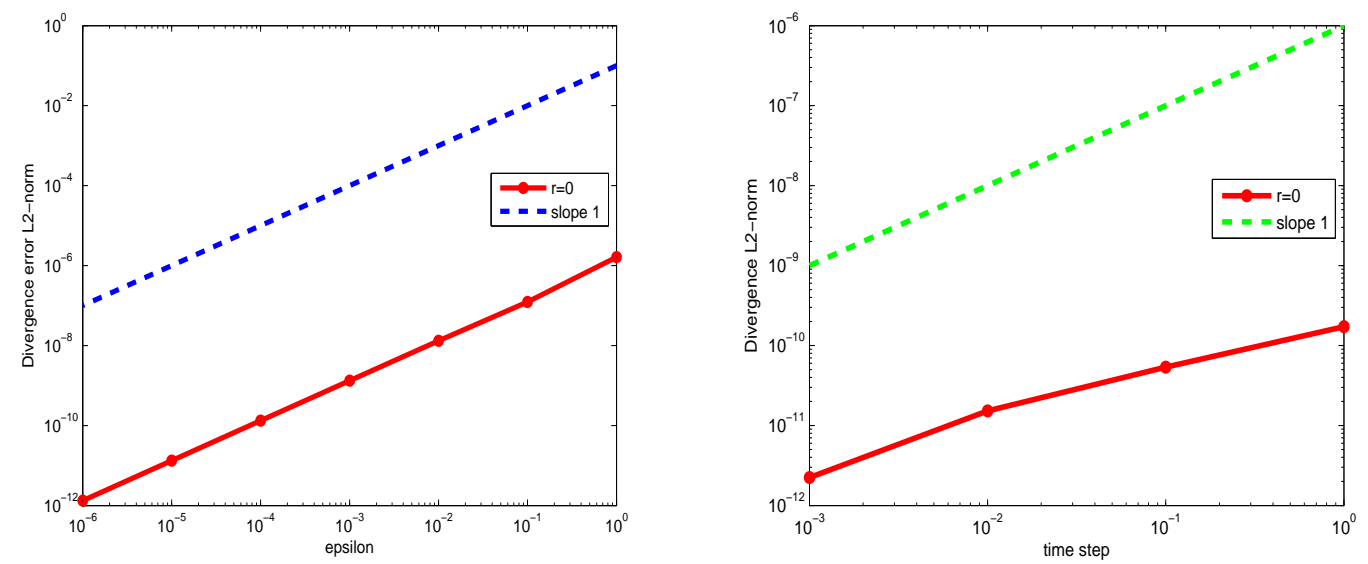

Figure 8: Homogeneous open boundary conditions. OBC2 - Velocity divergence $L^{2}$-norm versus $\varepsilon$ (left) at $T=2$, mesh size $1 / h=128$ and $r=0-$ Velocity divergence $L^{2}$-norm versus time step (right) at $T=2$, mesh size $1 / h=128$ and $\varepsilon=10^{-6}$.

Remark 5.2. We numerically check the value of $\widehat{\mathbf{v}} \cdot \mathbf{n}$ on the boundary $\Gamma_{N}$ in the vector penalty-projection step with OBC2 (4.26). We obtain that $\widehat{\mathbf{v}} \cdot \mathbf{n}$ is of order $10^{-12}$ which means that this condition is approximately satisfied naturally on $\Gamma_{N}$ in the projection step.

\section{Convergence rate in time at $r=0$ for the VPP methods with OBC1 (4.6) and the pressure gradient given by (4.10)}

In this section, we study the temporal convergence of the special case: $r=0$ and $\varepsilon$ too small. For this purpose, we use the same test as for the case of homogeneous open boundary conditions above. We study the method with the proposed open boundary condition OBC1 (4.6) on $\Gamma_{N}$ in the projection step. This study also allows a comparison with the case $10^{-4} \leq r \leq 1$ already tested in the previous section.

Fig. 9 (left) displays the errors of the computed velocity in the $L^{2}$-norm at $r=0$ and $\varepsilon=10^{-10}$. In contrast to the case $10^{-4} \leq r \leq 1$, we do not have a second-order convergence rate: the slope of the velocity error appears to be rather of first order. Moreover, we observe in Fig. 9 (right) a sharp degradation of the pressure convergence (order $1 / 2$ only). This degradation is due to the cumulation of round-off errors when we use the standard pressure-correction (4.8) with very small values of $\varepsilon$.

Using the pressure gradient correction (4.10) explained in Section 4.1.2, i.e, we repeat the same tests for the velocity and the pressure gradient for $r=0$. Fig. 10 (left) shows that we recover a second-order convergence rate in time for the velocity as in the case of $10^{-4} \leq r \leq 1$. In Fig. 10 (right), the pressure gradient reaches the order of $\mathcal{O}\left(\delta t^{1.8}\right)$. Finally, these positive results confirm the interest in updating the pressure by its gradient and are in a agreement with [1]. 

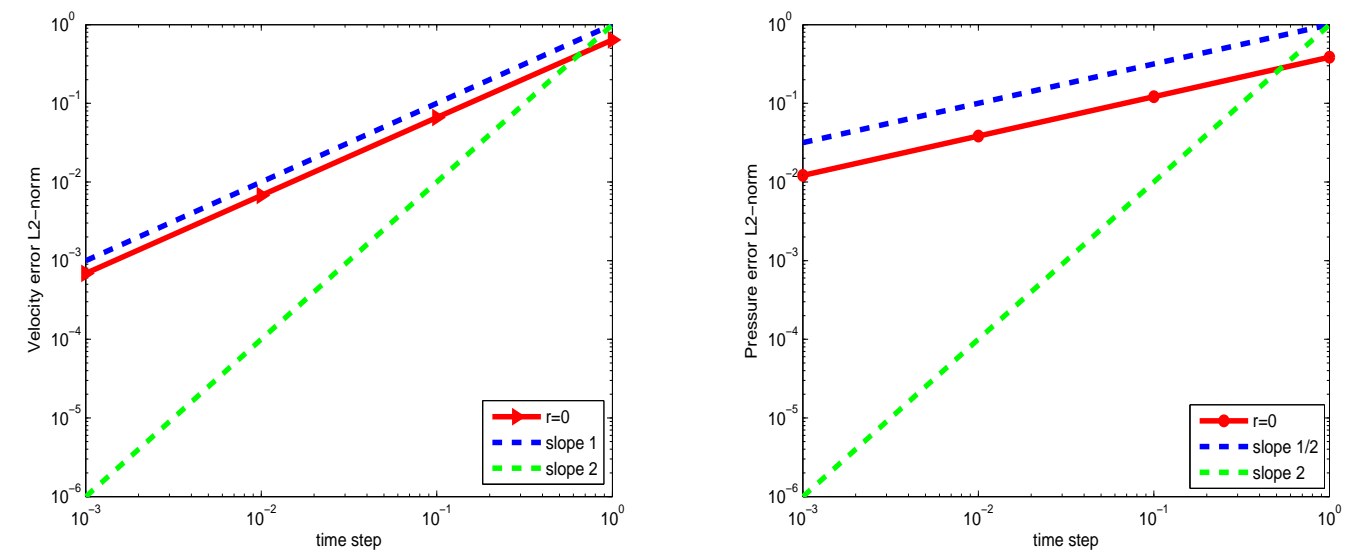

Figure 9: Homogeneous open boundary conditions. OBC1 - Velocity error $L^{2}$-norm (left) and pressure error $L^{2}$-norm (right) versus time step with the standard pressure correction (4.8) at mesh size $1 / h=128, r=0$ and $\varepsilon=10^{-10}$.
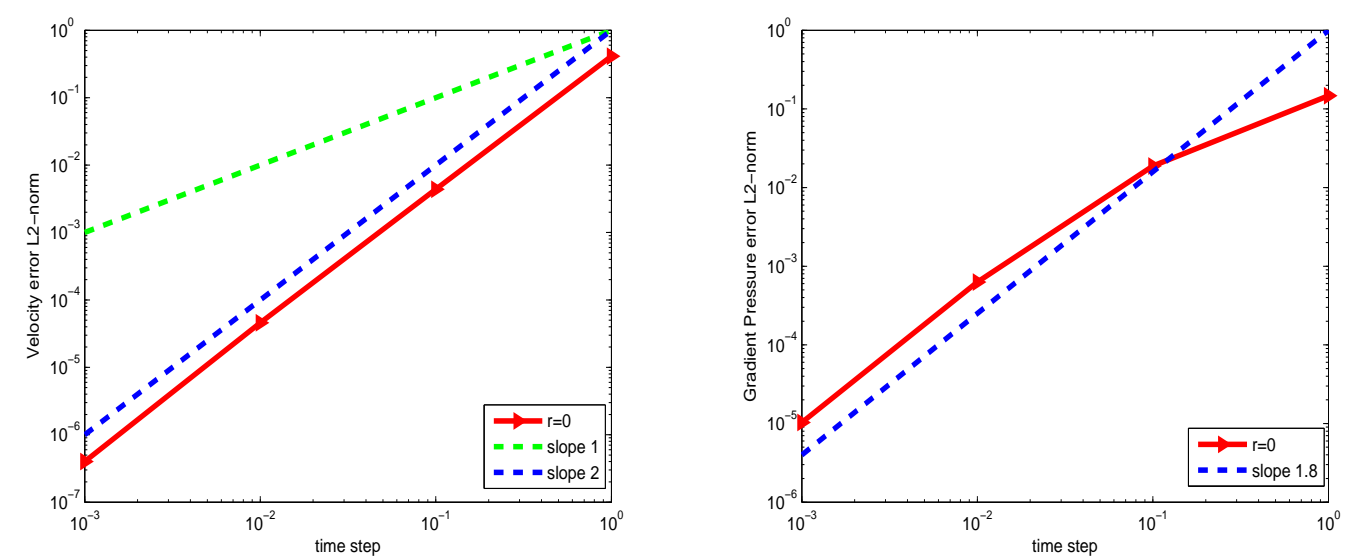

Figure 10: Homogeneous open boundary conditions. OBC1 $-L^{2}$-norm of the error for the velocity (left) and the pressure gradient (right) versus time step with the pressure gradient correction (4.10) at mesh size $1 / h=128$, $r=0$ and $\varepsilon=10^{-10}$.

\subsubsection{Nonhomogeneous open boundary conditions $(\mathrm{g} \neq 0)$}

To further assess the influence of open boundary conditions on the accuracy of BDF2VPP methods, we have performed temporal convergence tests for the nonhomogeneous case. To this end, we consider the same problem as in [32,33]:

$$
\begin{aligned}
& u(x, y, t)=\cos ^{2}\left(\frac{\pi x}{2}\right) \sin (\pi y) \cos (2 \pi \omega t), \\
& v(x, y, t)=-\cos ^{2}\left(\frac{\pi y}{2}\right) \sin (\pi x) \cos (2 \pi \omega t),
\end{aligned}
$$




$$
p(x, y, t)=\cos \left(\frac{\pi x}{2}\right) \sin \left(\frac{\pi y}{2}\right) \cos (2 \pi \omega t) .
$$

Convergence rate in time. We take a mesh size $1 / h=128$ and we suppose $\omega=1$. The representative curves of Fig. 11 show that the convergence rates of the error on the velocity and the pressure is of order 2 for an augmentation parameter $10^{-4} \leq r \leq 1$. This result is in line with the results reported in [33]. We observe that the errors are not very different from those computed with the homogeneous case studied above. Besides, the $L^{2}$-norm of the velocity divergence vanishes roughly as $\mathcal{O}(\varepsilon \delta t)$ for $\varepsilon$ too small (see Fig. 12).
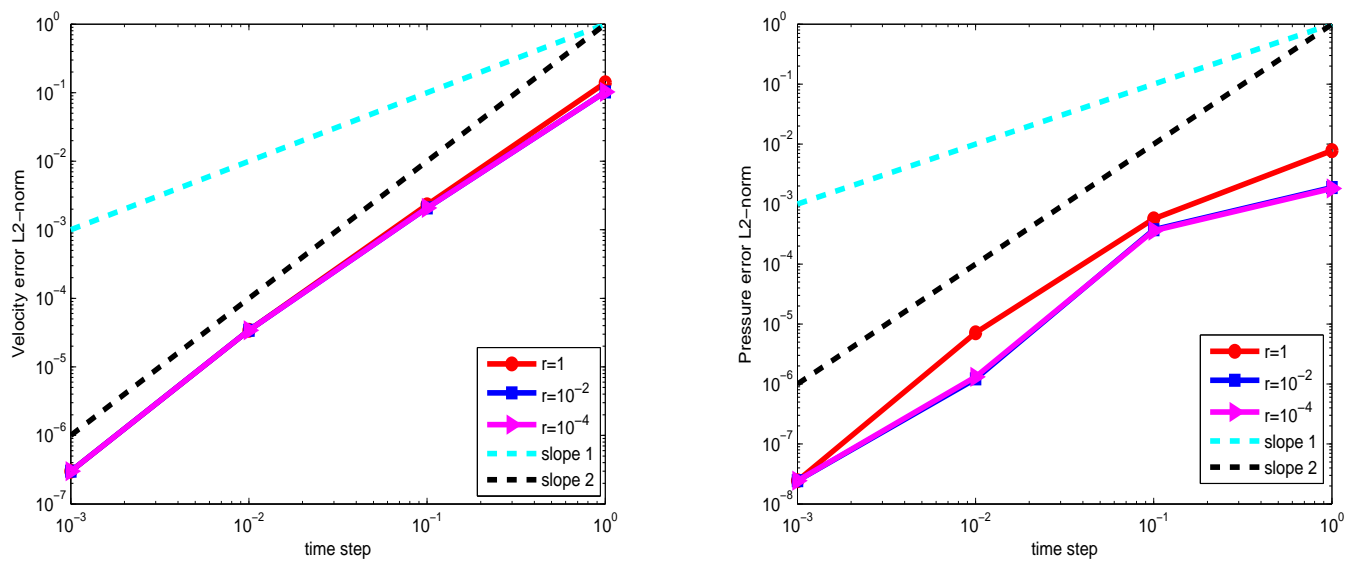

Figure 11: Nonhomogeneous open boundary conditions. OBC1 - Temporal convergence rates for the velocity (left) and the pressure (right) at $T=2$, mesh size $1 / h=128$ and $\varepsilon=10^{-10}$.
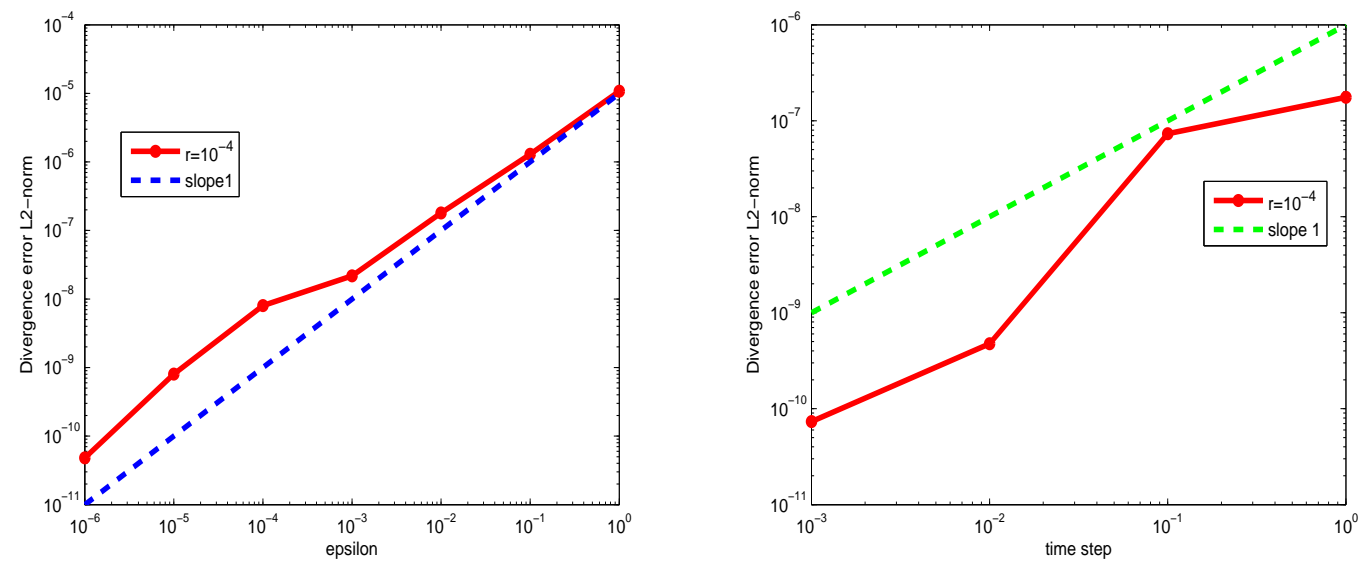

Figure 12: Nonhomogeneous open boundary conditions. OBC1 $-L^{2}$-norm of the divergence of the velocity versus $\varepsilon$ (left) at $T=2$, mesh size $1 / h=128$ and $r=10^{-4}-L^{2}$-norm of the velocity divergence versus time step (right) at $T=2$, mesh size $1 / h=128, \varepsilon=10^{-6}$. 

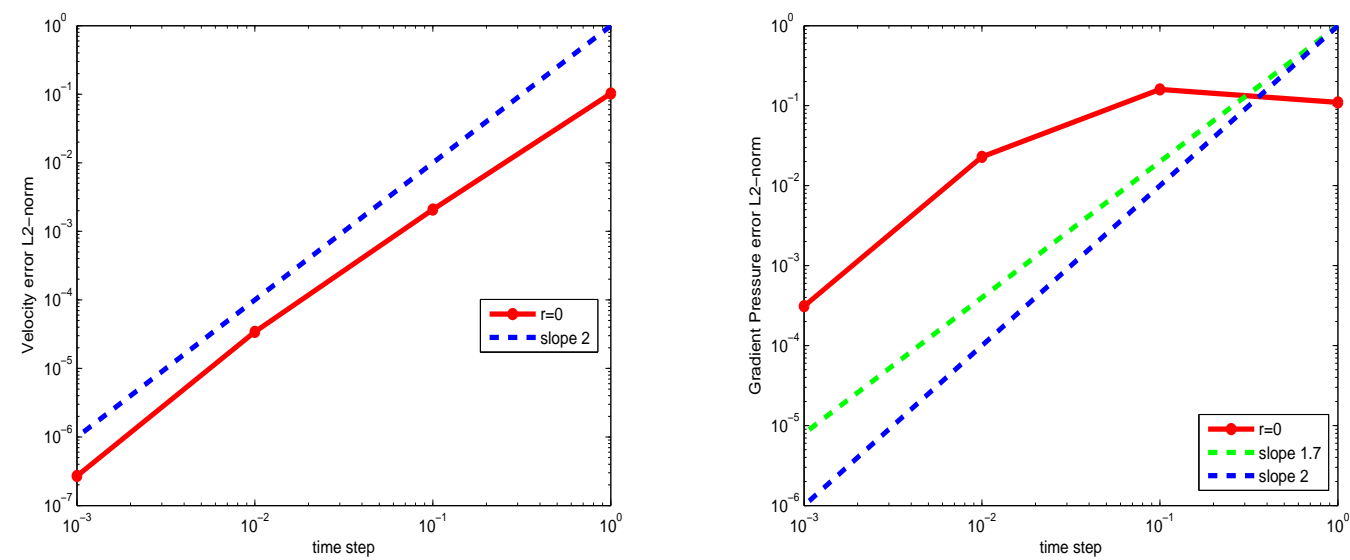

Figure 13: Nonhomogeneous open boundary conditions. OBC2 - Temporal convergence rates for the velocity (left) and the gradient pressure (right) at $T=2$, mesh size $1 / h=128, \varepsilon=10^{-10}$ and $r=0$.
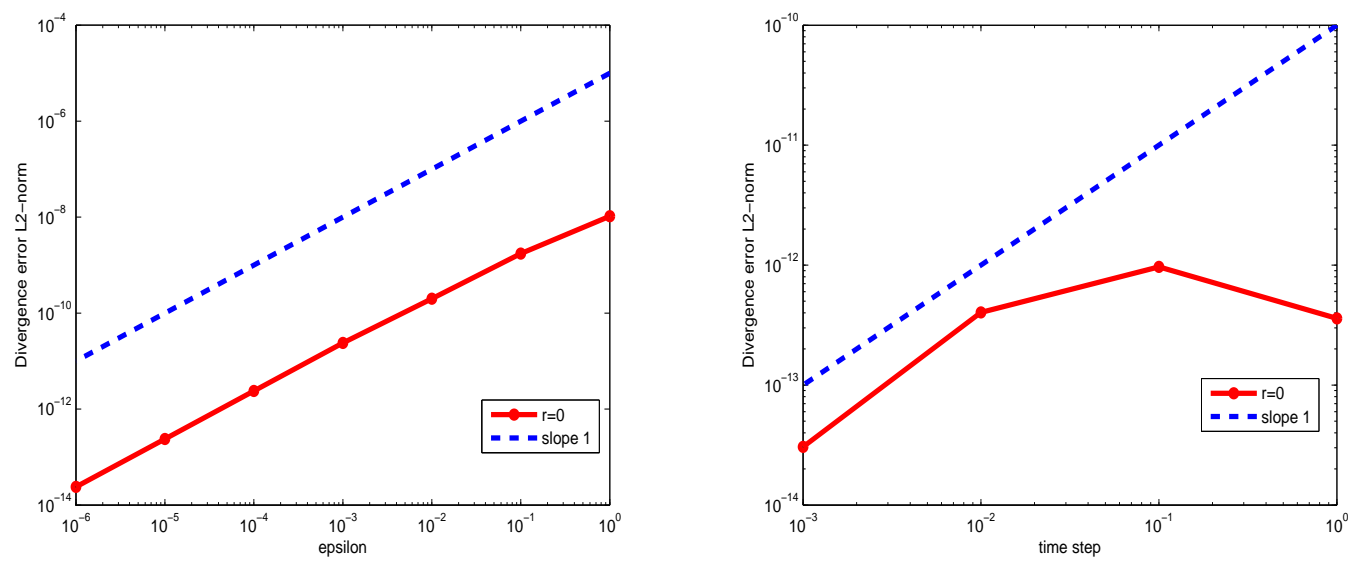

Figure 14: Nonhomogeneous open boundary conditions. OBC2 - Velocity divergence $L^{2}$-norm versus $\varepsilon$ (left) at $T=2$, mesh size $1 / h=128$ and $r=0$ - Velocity divergence $L^{2}$-norm versus time step (right) at $T=2$, mesh size $1 / h=128, \varepsilon=10^{-6}$ and $r=0$.

We now present the same test using the VPP methods with (OBC2). Let $r=0$ and $\varepsilon=10^{-10}$. As one can see in Fig. 13 (left), the convergence rate of the error on the velocity is clearly of order $\mathcal{O}\left(\delta t^{2}\right)$. Fig. 13 (right) shows the error on the pressure gradient measured in $L^{2}$-norm. The results reveal clearly that the pressure gradient approximation is roughly of order 1.7 in time. We note that the saturation observed here for small time steps is due to the approximation error in space which becomes dominant for very small time steps. In this case, the velocity divergence is almost of order $\mathcal{O}(\varepsilon \delta t)$ as $\varepsilon$ tends to zero (see Fig. 14). 


\subsection{Numerical results for Navier-Stokes problem}

In order to validate the accuracy of the method for the nonlinear Navier-Stokes equations, we present the temporal convergence studies on two manufactured test cases: first, with homogeneous open boundary conditions and second, with nonhomogeneous open boundary conditions. To this end, we consider the VPP methods with the open boundary condition OBC2 (4.26). For the approximation of the time derivative, the BDF2 scheme is used. The convective terms of the Navier-Stokes equations are treated explicitly then the second order central difference scheme is applied to its conservative form. This choice ensures overall second order accuracy. The finite volume scheme on a MAC staggered grid arrangement is adopted in order to remove the odd-even decoupling. Simulations are performed using a range of time steps $10^{-5} \leq \delta t \leq 5 \times 10^{-3}$ at $T=2$ with a Reynolds number $R e=100$. Note that $\delta t$ is chosen sufficiently small to satisfy the Courant-Friedrichs-Lewy condition (CFL condition). We choose $r=0$ and $\varepsilon=10^{-10}$. The linear systems obtained are solved with the preconditioned conjugate gradient CG-IC(0). To check the temporal accuracy, we carry out the following tests.

- For the homogeneous open boundary conditions $(g=0)$, the Navier-Stokes equations are augmented with a forcing term in order that the solution is

$$
\begin{aligned}
& u(x, y, t)=-\sin (\pi x) \cos (\pi y) \exp (-\mu t), \\
& v(x, y, t)=\cos (\pi x) \sin (\pi y) \exp (-\mu t) \\
& p(x, y, t)=-\mu \pi \cos (\pi x) \cos (\pi y) \exp (-\mu t) .
\end{aligned}
$$

- For the nonhomogeneous open boundary conditions $(\mathbf{g} \neq 0)$, the source term $\mathbf{f}$ is adjusted such that the Navier-Stokes problem verify the following problem

$$
\begin{aligned}
& u(x, y, t)=\cos ^{2}\left(\frac{\pi x}{2}\right) \sin (\pi y) \exp (-2 \pi \mu t), \\
& v(x, y, t)=-\cos ^{2}\left(\frac{\pi y}{2}\right) \sin (\pi x) \exp (-2 \pi \mu t), \\
& p(x, y, t)=\cos \left(\frac{\pi x}{2}\right) \sin \left(\frac{\pi y}{2}\right) \exp (-2 \pi \mu t) .
\end{aligned}
$$

Convergence rate in time. In Fig. 15 (left), we plot the $L^{2}$-norm error on the velocity and the pressure gradient as function of the time step $\delta t$ for the homogeneous test case. Errors are calculated at the time $T=2$ and for $r=0$ after computations on a square domain with the mesh size $h$ equal to $1 / 128$. As expected, the nonlinear term in the NavierStokes equations does not really affect the convergence rate for both the velocity and the pressure gradient. We obtain an order of 1.85 in time for both unknowns. For the nonhomogeneous case, the temporal error of the velocity and the pressure gradient computed on $128 \times 128$ grids are plotted in Fig. 15 (right). The results reveal an order of 1.85 in time for both unknowns. The accuracy saturation observed for small time steps results from the spatial discretization error. 

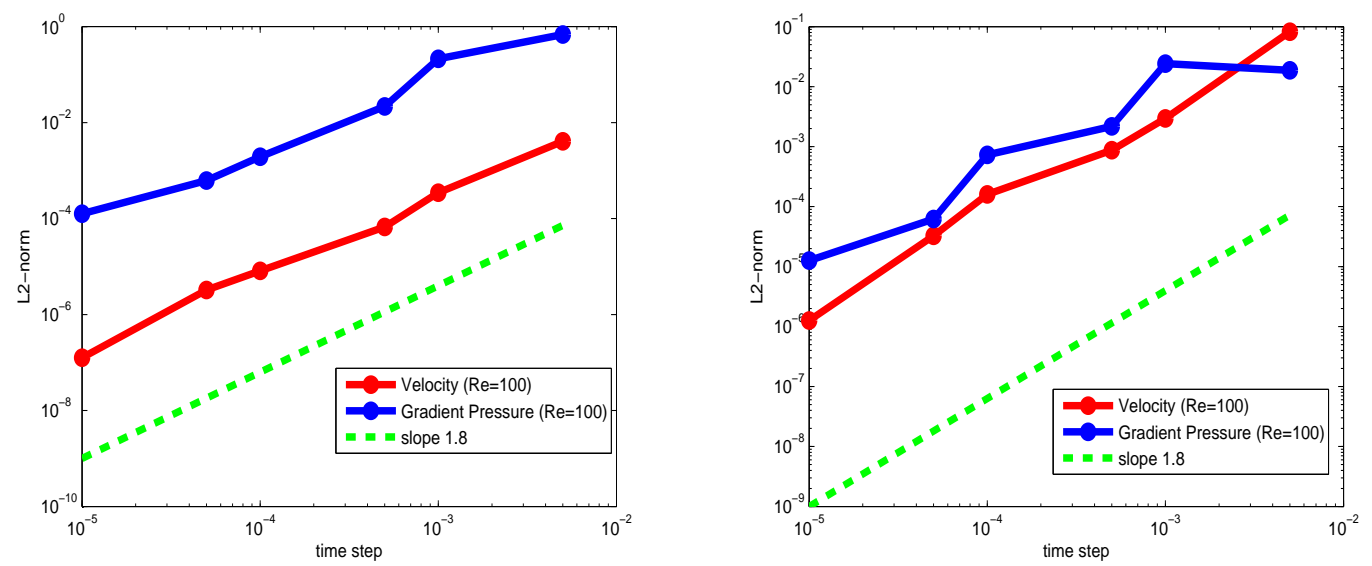

Figure 15: Homogeneous (left) and nonhomogeneous (right) open boundary conditions. OBC2 $-L^{2}$-norm of the error for the velocity and the pressure gradient versus $\delta t$ at $T=2$ and $R e=100$, mesh size: $1 / h=128$, $\varepsilon=10^{-6}, r=0$ for Navier-Stokes problem.

In conclusion, we deduce that for the Navier-Stokes problem, the nonlinear term does not really damage the convergence rate. However, we do not have an optimal convergence of second-order in time.

\subsection{Summary of the numerical results and discussion}

We summarize in this section the advantages of the VPP methods.

1. The open boundary conditions were naturally extended on the boundary $\Gamma_{N}$. Consequently, as shown numerically in the above sections, the velocity and the pressure (or the pressure gradient) reach approximately a second order convergence rate in space and time even in the presence of the open boundary conditions.

2. The nonlinear terms in the Navier-Stokes problem does not affect the convergence rate in time for the velocity and the pressure. We note that the convergence rate is slightly degraded but we obtain approximately a second order in time.

3. The VPP methods appear fast, cheap and require only few iterations for small penalty parameter $\varepsilon$.

\section{Conclusions}

In this article, we have naturally extended the VPP methods to the case of incompressible viscous flows with open boundary conditions using two kinds of second-order schemes for time discretization: the BDF2 scheme and the Crank-Nicolson scheme. The numerical 
experiments show that the VPP methods yield a considerable gain in accuracy compared to the pressure-correction schemes [19]. The convergence rate in time for velocity and pressure obtained using VPP methods are also in agreement with those obtained by the standard and rotational form of the velocity-correction method with a proposed boundary condition [34]. Moreover, we show that for both second-order schemes used for time discretization (BDF2 or Crank-Nicolson), the VPP methods yield approximately $\mathcal{O}\left(\delta t^{2}\right)$ for both the velocity and the pressure for the homogeneous as well as and nonhomogeneous open boundary conditions. The loss of spatial convergence in the case of open boundary conditions does not occur anymore. We obtain $\mathcal{O}\left(h^{2}\right)$ convergence in the $L^{2}$ norm of the velocity and the pressure. Indeed, the optimal second-order accuracy in time and space is achieved because of the fully vector formulation of the VPP methods, without degrading it by a scalar pressure Poisson equation. This inherently ensures the consistency with respect to the continuous Navier-Stokes problem with a traction boundary condition. The counterpart is that the divergence of the velocity at each time step is not exactly zero, as for the projection methods (at least in the semi-discrete setting in time), since the VPP velocity correction step is proved to be an approximate divergencefree projection $[1,9]$. However, it is not really a drawback since the velocity divergence is in practice of order $\mathcal{O}(\varepsilon \delta t)$ with a penalty parameter $\varepsilon$ taken as small as desired up to machine precision. Finally, the method proves to be very efficient: it is fast, cheap, and provides very accurate results with optimal spatial and temporal convergence rates despite the existence of open boundary conditions. Moreover, this family of methods opens the way to the splitting methods with an order of time convergence greater than 2 since the splitting error for velocity and pressure varies as $\mathcal{O}(\varepsilon)$ which can be made negligible with respect to the consistency error of higher-order schemes when $\varepsilon$ is chosen sufficiently small.

\section{References}

[1] Ph. Angot, J.-P. Caltagirone, and P. Fabrie, Analysis for the fast vector penaltyprojection solver of incompressible multiphase Navier-Stokes/brinkman problems, Numer. Math.(2016), submitted for publication.

[2] Ph. Angot and R. Cheaytou, Vector penalty-projection methods for incompressible fluid flows with open boundary conditions, in Algoritmy 2012- (A. Handlovicočá et al. Eds), Slovak University of Technology in Bratislava, Publishing House of STU (Bratislava) (2012), 219-229.

[3] Ph. Angot and R. Cheaytou, On the error estimates of the vector penalty-projection methods: Second-order scheme, Math. Comp., 87 (2018), 2159-2187

[4] Ph. Angot, J.-P. Caltagirone and P. Fabrie, Vector penalty-projection methods for the solution of unsteady incompressible flows, Finite Volumes for Complex Applications V, (eds R. Eymard and J.-M. Herard), ISTE Ltd and J. Wiley and Sons, 59 (2008), 169-176.

[5] Ph. Angot, J.-P. Caltagirone and P. Fabrie, A new fast method to compute saddle-points in constrained optimization and applications, Appl. Math. Lett., 25 (2012), no. 3, 245-251.

[6] Ph. Angot, J.-P. Caltagirone and P. Fabrie, A fast vector penalty-projection method for incompressible non-homogeneous or multiphase Navier-Stokes problems, Appl. Math. Lett., 
25 (2012), no. 11, 1681-1688.

[7] Ph. Angot, J.-P. Caltagirone and P. Fabrie, A spectacular vector penalty-projection method for Darcy and Navier-Stokes problems, in, J. Fort, et al.(Eds.), Finite Volumes for Complex Applications VI- Problems and Perspectives, in: Springer Proceedings in Mathematics, 1, no. 4, Springer-Verglas, Berlin, (2011).

[8] Ph. Angot, M. Jobelin, and J.-C. Latche, Error analysis of the penalty-projection method for the time dependent Stokes equations, Int. J. Finite Vol. 6 (2009), no. 1, 1-26.

[9] Ph. Angot and J.-P. Caltagirone and P. Fabrie, Fast discrete Helmholtz-Hodge decompositions in bounded domains, Appl. Math. Lett., 26 (2013), no. 4, 445-451.

[10] Ph. Angot, On the unsteady Stokes problem with a nonlinear open artificial boundary condition modelling a singular load, (2013), ¡hal-00820404i.

[11] Ph. Angot and P. Fabrie, Convergence results for the vector penalty-projection and two-step artificial compressibility methods, Discrete Contin. Dyn. Syst. Ser. B, 17 (2012), no. 5, 13831405.

[12] J.-P. Caltagirone and J. Breil, Sur une méthode de projection vectorielle pour la résolution des équations de Navier-Stokes, C. R. Math. Acad. Sci. Paris, 327 (1999), no. 11, 1179-1184.

[13] R. Cheaytou, Etude des méthodes de pénalité-projection vectorielle pour les équations de navier-stokes avec conditions aux limites ouvertes, Ph.D. thesis, Université d'Aix-Marseille, April 2014.

[14] A.J. Chorin, Numerical solution of the Navier-Stokes equations, Math. Comp., 22 (1968), 745-762.

[15] J. Crank and P. Nicolson, A practical method for numerical evaluation of solution of partial differential equations of the heat-conduction type, Proc. Cambridge Philos. Soc., 43 (1947), $50-67$.

[16] C. Févrière, J. Laminie, P. Poullet and Ph. Angot, On the penalty-projection method for the Navier-Stokes equations with the MAC mesh, J. Comput. Appl. Math., 226 (2009), 228-245.

[17] M. Fortin and R. Glowinski, Augmented lagrangians: Application to the numerical solution of boundary value problems, Ed. North-Holland, Amsterdam (1983).

[18] K. Goda, A multistep technique with implicit difference schemes for calculating two- or three-dimensional cavity flows, J. Comput. Phys., 30 (1979), no. 1, 76-95.

[19] J.L. Guermond and J. Shen, On the error estimates for the rotational pressure-correction projection methods, Math. Comp., 73 (2004), no. 248, 1719-1737.

[20] J.L. Guermond, P. Minev and J. Shen, An overview of projection methods for incompressible flows, Comput. Methods Appl. Mech. Engrg., 195 (2006), 6011-6045.

[21] J.L. Guermond, P. Minev and J. Shen, Error analysis of pressure-correction schemes for the time-dependent Stokes equations with open boundary conditions, SIAM J. Numer. Anal., 43 (2005), 239-258.

[22] F. Harlow and J. Welch, Numerical calculation of time dependent viscous incompressible flow of fluid with free surface, J. Eur. Phys. Fluids, 8 (1965), no. 12, 2182-2189.

[23] N. Hasan and S. Anwer and S. Sanghi, On the outflow boundary condition for external incompressible flows: A new approach, J. Comput. Phys., 206 (2005), no. 2, 661-683.

[24] He Yinnian, Two-level method based on finite element and Crank-Nicolson extrapolation for the time-dependent Navier-Stokes equations, SIAM J. Numer. Anal. 41 (2003), no. 4, 1263-1285.

[25] He Yinnian and Liu Kam-Moon, A multilevel finite element method in space-time for the Navier-Stokes problem. Numer. Methods for Partial Differential Equations, 21 (2005), no. 6, 1052-1078. 
[26] He Yinnian and Sun Weiwei, Stabilized finite element method based on the Crank-Nicolson extrapolation scheme for the time-dependent Navier-Stokes equations, Math. Comp. 76 (2007), no. 257, 115-136.

[27] He Yinnian and Sun Weiwei Stability and convergence of the Crank-Nicolson/AdamsBashforth scheme for the time-dependent Navier-Stokes equations, SIAM J. Numer. Anal. 45 (2007), no. 2, 837-869.

[28] He, Yinnian, The Euler implicit/explicit scheme for the 2D time-dependent Navier-Stokes equation with smooth or non-smooth initial data. Math. Comp., 77 (2008), no. 264, 20972124.

[29] S. M. Hosseini and J. J. Feng, Pressure boundary conditions for computing incompressible flows with SPH, J. Comput. Phys., 23 (2011), no. 19, 7473-7487.

[30] M. Jobelin and C. Lapuerta and J.C Latché and Ph. Angot and B. Piar, A finite element penalty-projection method for incompressible flows, J. Comput. Phys., 217 (2006), 502-518.

[31] Kh. Khadra and Ph. Angot and S. Parneix and J.P. Caltagirone, Fictitious domain approach for numerical modelling of Navier-Stokes equations, Internat. J. Numer. Methods Fluids, 34 (2000), no. 8, 651-684.

[32] J. Liu, Open and traction boundary conditions for the incompressible Navier-Stokes equations, J. Comput. Phys., 228 (2009), 7250-7267.

[33] A. Poux and S. Glockner and M. Azaïez, Improvements on open and traction boundary conditions for Navier-Stokes time-splitting methods, J. Comput. Phys., 230 (2011), 4011-4027.

[34] A. Poux, S. Glockner, E. Ahusborde, and M. Azaïez, Open boundary conditions for the velocity-correction scheme of the NavierStokes equations, Comput. Fluids, 73 (2012), no. 70, 29-43.

[35] J. Shen, On error estimates of some higher order projection and penalty-projection methods for Navier-Stokes equations, Numer. Math., 62 (1992), 49-74.

[36] R. Temam, Sur l'approximation de la solution des équations de Navier-Stokes par la méthode des pas fractionnaires (II), Arch. Rational Mech. Anal., 33 (1969), 377-385.

[37] L.J.P. Timmermans, P.D. Minev, and F.N. Van De Vosse, An approximate projection scheme for incompressible flow using spectral elements, Internat. J. Numer. Methods Fluids, 22 (1996), no. 7, 673-688.

[38] J.Van Kan, A second-order accurate pressure-correction scheme for viscous incompressible flow, SIAM J. Sci. Comput., 7 (1986), no. 3, 870-891. 